\title{
LEGITTIMITÀ, CONSOLIDAMENTO E CRISI NELL'EUROPA MERIDIONALE
}

\author{
di Leonardo Morlino e José Ramon Montero
}

Nei primi anni novanta gli effetti politici della crisi economica si fanno sentire in tutti i paesi occidentali, e soprattutto in quelli caratterizzati da economie più deboli, come i paesi del Sud Europa. Crescono gli atteggiamenti di critica e protesta, e in questo senso diminuisce la legittimità governativa. Tutto ciò si manifesta in Grecia, innanzi tutto, con la sconfitta del governo conservatore nelle elezioni dell'ottobre 1993, in Spagna con la diminuzione dei voti socialisti nel giugno dello stesso anno e la perdita della maggioranza assoluta in parlamento, in Portogallo con più forti dissensi e critiche al governo in assenza di elezioni. In Italia i fenomeni di protesta sono più profondi, al punto di poter parlare - in questo stesso periodo - di crisi e transizione di regime. L'Italia, dunque, presenta un «differenziale» rispetto agli altri paesi che va spiegato ${ }^{1}$

Tra i diversi fattori esplicativi, quello su cui si è finora riflettuto di meno riguarda proprio le diverse caratteristiche che la legittimità presenta in Italia rispetto agli altri paesi. Come sarebbe possibile, infatti, che una società civile che ancora a metà degli anni ottanta esibisce alti livelli di legittimità, dopo qualche anno affronti la maggiore crisi dopo il 1945 e inizi un mutamento nelle principali strutture democratiche, dal sistema parti-

Una prima versione di questo saggio ha tratto grande beneficio dai commenti dei componenti del Subcommittee on Southern Europe del Social Science Research Council (USA). Desideriamo qui ringraziarli vivamente per le loro osservazioni e critiche. Un particolare ringraziamento va anche a Franco Mattei e Mariano Torcal per il loro prezioso aiuto nell'elaborazione dei dati. Infine, a Giacomo Sani e Julián Santamaria vorremmo esprimere la nostra gratitudine per come banno saputo affrontare tutti $i$ numerosi e complessi problemi che comporta la direzione di un ampio sondaggio su quattro paesi, per $i$ cui dettagli si rimanda alla nota 2.

1 Sulla crisi vedi Crozier et al. (1975); sull'ingovernabilità vedi Rose (1980); sul sovraccarico vedi King (1975).

RIVISTA ITALIANA DI SCIENZA POLITICA / a. XXIV, n. 1, aprile 1994 
tico a quello elettorale? In questo articolo, dunque, si comincia con il definire meglio in che misura le democrazie del Sud Europa erano considerate legittime a metà degli anni ottanta; e, poi, quali siano le principali caratteristiche di questa legittimità che aiutano a spiegare sia i problemi più seri dell'Italia che quelli meno gravi degli altri tre paesi dell'Europa meridionale. Iniziamo, però con una disamina del concetto di legittimità, la quale serve da introduzione teorica alla successiva analisi dei dati. Verranno poi prese in esame le distribuzioni degli atteggiamenti nell'opinione pubblica dell'Europa del Sud verso i regimi democratici, le esperienze autoritarie del passato e la soddisfazione per il funzionamento della democrazia, nonché alcune possibili spiegazioni di questi risultati. L'analisi verrà poi ampliata, e verranno esaminati altri orientamenti rilevanti, specie verso la politica in generale. Infine, dato che focalizziamo l'attenzione sulla legittimità democratica, verrà trattata una domanda ovvia: legittimazione di cosa? In altre parole, esamineremo le diverse concezioni di democrazia presso gli europei del Sud. I dati empirici analizzati sono stati raccolti nell'ambito del Four Nation Survey eseguito in Portogallo, Spagna, Italia e Grecia a metà degli anni ottanta $a^{2}$.

\section{La legittimità ed altri atteggiamenti positivi}

In questa sede per legittimità si intende un insieme di atteggiamenti favorevoli che una società esprime nei confronti delle proprie istituzioni democratiche, le quali vengono considerate la migliore forma di governo (cfr. Morlino 1980) ${ }^{3}$. Benché vi siano diverse definizioni alternative del concetto, quella appena proposta è condivisa da Lipset (1959), Almond e Verba (1963),

\footnotetext{
2 Il sondaggio, diretto da Julián Santamaria e Giacomo Sani, è stato effettuato nella primavera del 1985. Complessivamente furono intervistate 8570 persone: 2000 dalla Norma in Portogallo; 2488 dal Centro de Investigaciones Sociológicas in Spagna; 2074 dalla Doxa in Italia; e 1998 dal Centre of Social Research in Grecia. Il gruppo di ricerca portoghese comprende Mario Bacalhau e Maria José Stock; quello spagnolo Rosa Conde, Ubaldo Martínez, José R. Montero e Julián Santamaria; quello italiano Giovanna Guidorossi, Renato Mannheimer, Leonardo Morlino, Giacomo Sani e Maria Weber; e quello greco George Th. Mavrogordatos, Ilias Nicolacopoulos e Constantinos Tsoucalas.

${ }^{3}$ In questa sede ci riferiamo alla legittimità politica, e l'analisi si limiterà a questo aspetto. Sarebbe possibile, tuttavia, aggiungere due altre dimensioni: la legittimità sociale e quella economica (cfr. Linz 1988).
} 
Linz (1978), nonché da molti altri autori. Lo sviluppo di atteggiamenti positivi verso la democrazia è un aspetto importante del consolidamento democratico, in quanto essi incoraggiano comportamenti di lealtà e obbedienza.

I nostri sondaggi, condotti nell'Europa del Sud a metà degli anni ottanta, forniscono indizi empirici su questo punto. In tre dei quattro paesi studiati i sondaggi venivano svolti dopo l'instaurazione di un regime democratico, verso il quale cominciavano a formarsi i primi atteggiamenti di massa. Nelle prime fasi della democratizzazione non è ragionevole aspettarsi di trovare un forte radicamento degli atteggiamenti di sostegno al sistema, né dei comportamenti ad essi connessi. Anche se possono essere diffusi orientamenti vagamente favorevoli alla democrazia, il sostegno per uno specifico regime democratico e per le sue istituzioni centrali non può aver avuto modo di svilupparsi. Questi atteggiamenti si rafforzano progressivamente nel periodo di consolidamento. Di conseguenza, gli studiosi che sottolineano l'importanza della legittimità ritengono che un regime democratico sia pienamente consolidato solo quando si raggiunge un elevato grado di legittimità.

In alcuni casi gli atteggiamenti positivi si sviluppano rapidamente, mentre in altri il processo di legittimazione dura più a lungo: può richiedere anni. Questo processo, peraltro, non è necessariamente unilineare: può essere interrotto da crisi o addirittura subire un'inversione di tendenza. Come abbiamo accennato in precedenza, è proprio a quest'ultima fattispecie che può essere ricondotto il caso italiano.

Come si dovrebbe misurare la legittimità? Gli studiosi hanno posto questa classica domanda in modi molti diversi, a volte con intenti polemici. Per alcuni la legittimità è un concetto carico di valore, la cui stessa natura preclude l'analisi mediante indicatori convenzionali; per altri è pressoché impossibile coglierla in modo empirico (Schaar 1981). Se si tiene conto della multidimensionalità del concetto, si complicano ulteriormente i problemi di misurazione e operativizzazione (McDonough et al. 1986). Inoltre, gli studi relativi a questo concetto e ad altri connessi sono caratterizzati da un notevole divario tra un grado relativamente elevato di articolazione teorica e concettuale, da un lato e, dall'altro, la debolezza dei numerosi indicatori empirici finora impiegati. Infine, l'analisi del concetto viene ulteriormente complicata dal fatto che un regime non è mai del tutto legittimo per tutti, né gode dello stesso grado di legittimità o di sostegno presso ogni settore sociale. 
Dinanzi a una complessità così scoraggiante, abbiamo adottato un'accezione piuttosto relativa e minimalista: la legittimità è definita dalla convinzione che, nonostante $\mathrm{i}$ difetti e i fallimenti, le istituzioni politiche esistenti sono migliori delle altre che potrebbero venire introdotte ( $\operatorname{Linz} 1978 ; 1988)$; ovvero dalla convinzione che un regime politico è ritenuto il «meno peggiore» fra tutte le possibili forme di governo. Come ha scritto Linz (1968), «in ultima analisi, la legittimità democratica si basa sulla credenza che, per un determinato paese in un particolare momento, nessun altro tipo di regime sarebbe in grado di garantire meglio la realizzazione degli obiettivi collettivi».

Così il nostro approccio richiede l'analisi empirica di due distinte dimensioni della legittimità e di un terzo insieme di atteggiamenti, empiricamente e teoricamente collegato ma concettualmente diverso dalle prime due. In primo luogo, prendiamo in considerazione gli atteggiamenti più generali e astratti relativi a un regime democratico: la democrazia è preferibile a qualsiasi altro tipo di regime? Possiamo chiamare «legittimità diffusa» gli atteggiamenti generali di questo tipo (Morlino 1980). Questi atteggiamenti poggiano sulle opinioni circa la democrazia come tipo di regime, per cui la loro salienza è maggiore nei paesi che hanno avuto esperienze di altri regimi (autoritari o totalitari). Ciò è vero per tutti e quattro i paesi europeomeridionali, i cui cittadini hanno conosciuto un regime antidemocratico di person $a^{4}$ o mediante la memoria collettiva sedimentata nella cultura. Per tale motivo, agli occhi di questi cittadini le risposte alle domande di un lungo sondaggio su questa dimensione sono molto più imbarazzanti, coinvolgenti e salienti che per cittadini di paesi che non hanno una storia recente di governo autoritario o totalitario.

La seconda dimensione della legittimità democratica di cui ci occuperemo attiene al grado in cui i cittadini di un regime democratico concepiscono l'esistenza di alternative preferibili al proprio sistema. Questa dimensione assume maggiore rilevanza in alcuni casi, come in Spagna e (in misura minore) in Portogallo, dove a una lunga esperienza autoritaria nel passato si sono

${ }^{4}$ In Italia coloro che al momento del Four Nation Survey del 1985 avevano avuto un'esperienza personale del vecchio regime autoritario da adulti o da adolescenti politicamente attivi (almeno sedicenni) avevano almeno 58 anni. In Grecia e Portogallo un'esperienza analoga interessava tutti coloro con più di 27 anni. In Spagna, anche i 24 enni avevano avuto un'esperienza diretta di un regime autoritario. 
accompagnati importanti processi di sviluppo economico e di modernizzazione sociale ed economica. Può essere di rilievo anche in casi, come quelli spagnolo e italiano, in cui la memoria collettiva di guerre e di altri generi di sofferenza politicamente rilevanti viene meno col passare del tempo e dove il ricordo dell'autoritarismo, specie tra gli anziani, può intrecciarsi con le piacevoli memorie legate alla gioventù.

Se le alternative al nuovo regime democratico non vengono giudicate positivamente, anche in presenza di valutazioni non necessariamente negative del passato autoritario, gli atteggiamenti di legittimità diffusa vengono rafforzati e dotati di una salienza aggiuntiva; il che rende più robusto il sostegno per il regime democratico. Potremmo chiamare questa seconda dimensione «legittimità per difetto di alternative». In alcuni casi, questa dimensione si può manifestare come una «legittimità per reazione contro il passato»s.

Quando si esaminano gli atteggiamenti favorevoli verso una democrazia, è opportuno tener conto anche della sua «efficacia percepita». È ragionevole aspettarsi che la soddisfazione di esigenze fondamentali mediante specifiche azioni di governo possa indurre la gente a maturare o rafforzare atteggiamenti positivi verso un regime democratico. La percezione diffusa della capacità di un sistema politico di risolvere i problemi è particolarmente importante nelle nuove democrazie, in cui tali atteggiamenti di sostegno al sistema possono non avere ancora profonde radici nella società. Perciò l'efficacia percepita di un regime appartiene a quell'insieme di atteggiamenti che è alla base della legittimità democratica e del riconoscimento delle istituzioni democratiche quale migliore forma di governo ${ }^{6}$.

$\grave{E}$ evidente che, presa in sé, in termini comportamentali l'efficacia può anche non influire sulla legittimità, come hanno rilevato per primi Lipset (1959) e Linz (1978; 1989). Da un altro punto di vista, alcuni studiosi hanno messo in dubbio la possibilità di distinguere sul piano empirico la legittimità dall'efficacia (Muller e Jukam 1977). Le elevate correlazioni che spesso si riscontrano suggeriscono che una struttura causale di tipo inde-

5 Le seguenti osservazioni sono state suggerite da alcuni lettori della versione preliminare di questo saggio, in particolare da Richard Gunther. Si vedano anche Di Palma (1990) e Linz e Stepan (1993).

6 Dahl (1971) attribuisce molta importanza alla percezione dell'efficacia. Va sottolineato che ci riferiamo all'efficacia del regime democratico, non alla percezione della «efficacia personale» di cui ci occuperemo più avanti. 
terminato colleghi i due concetti (Loewenberg 1971). Secondo questa argomentazione, le opinioni sulla legittimità di un regime sono da mettere in relazione con i giudizi circa i meriti delle autorità attualmente in carica, con le percezioni delle prestazioni istituzionali e/o con il divario tra ideali dei cittadini e realtà politica.

Noi crediamo che sia possibile distinguere analiticamente tra legittimità ed efficacia per una pluralità di ragioni. Il primo, e più importante, motivo si basa sulle esperienze non democratiche degli europei meridionali, anche se esse si riferiscono a periodi diversi e hanno avuto durata e intensità differenti. Queste esperienze personali di regimi di tipo diverso ci aiutano a distinguere empiricamente tra legittimità ed efficacia, in quanto greci, portoghesi, spagnoli e italiani possiedono quadri di riferimento precisi quando li si interroga circa le alternative politiche alla democrazia, la desiderabilità della democrazia in termini astratti e le prestazioni del regime.

Grazie alle loro esperienze personali o alla loro memoria collettiva, gli europei del Sud sono ben attrezzati culturalmente per poter distinguere tra legittimità di un regime e percezione della sua efficacia - anche se ci dobbiamo aspettare che questa capacità di distinguere venga gradualmente meno via via che, col passare del tempo, l'esperienza autoritaria perde il suo rilievo nella memoria collettiva del paese. Tuttavia, negli anni ottanta questa memoria era ancora viva e saliente, anche se in misura minore in Italia rispetto agli altri tre casi. Quando vengono intervistati i cittadini di democrazie di lunga data, privi di un passato non democratico, qualsiasi domanda sulle alternative politiche alla democrazia risulta sostanzialmente astratta e irrealistica. In mancanza di una concezione significativa delle alternative politiche, è probabile che la gente faccia riferimento all'efficacia e al rendimento quando le viene chiesto di dare un giudizio sulla legittimità.

\section{Livelli di legittimità e di efficacia}

Il Four Nation Survey prevedeva tre quesiti direttamente attinenti alle dimensioni della legittimità e dell'efficacia ${ }^{7}$. La tab.

7 I tre quesiti del sondaggio erano formulati come segue. Per la legittimità diffusa: «Con quali delle seguenti frasi si trova d'accordo? 1) La democrazia è preferibile a qua- 
TAв. 1. Atteggiamenti verso la democrazia nell'Europa del Sud, 1985 (percentuali di colonna)

\begin{tabular}{lllll}
\hline Portogallo & Spagna & Italia & Grecia \\
\hline
\end{tabular}

Legittimità diffusa

La democrazia è preferibile

L'autoritarismo è preferibile

$\grave{E}$ indifferente

Non so/nessuna risposta

Opinione sul passato autoritario

L'autoritarismo era una brutta cosa

In parte buono, in parte brutto

L'autoritarismo era una buona cosa

Non so/nessuna risposta

\section{Efficacia percepita}

La democrazia funziona bene

Funziona, anche se ha difetti

Sta peggiorando e non funzionerà

Non so/nessuna risposta

(N)

$\begin{array}{rrrr}61 & 70 & 70 & 87 \\ 9 & 10 & 13 & 5 \\ 7 & 9 & 10 & 6 \\ 23 & 11 & 7 & 2\end{array}$

$\begin{array}{rrrr}30 & 28 & 37 & 59 \\ 42 & 44 & 43 & 31 \\ 13 & 17 & 6 & 6 \\ 15 & 11 & 14 & 4\end{array}$

1 riporta le distribuzioni degli atteggiamenti verso questi concetti nei quattro paesi. Ovunque vi è un elevato livello di favore per la democrazia: più specificamente, due persone su tre esprimono una preferenza per la democrazia, mentre una su dieci preferirebbe un regime autoritario: due terzi degli intervistati pensano che la democrazia funzioni; soltanto una piccola minoranza giudica positivamente l'esperienza autoritaria del passato ${ }^{8}$.

$\grave{E}$ ancora più interessante notare le differenze tra i quattro paesi. In Italia sono più diffuse le posizioni critiche sulla legitti-

lunque altro regime; 2) In alcuni casi un regime autoritario, una dittatura, è preferibile; 3) Per gente come me non fa alcuna differenza». Per la legittimità per difetto, ovvero le opinioni sul passato: «Sulla base di quello che si ricorda del salazarismo (o franchismo, fascismo o dittatura) lei ritiene che: 1) fosse una cosa in parte buona e in parte brutta; 2) fosse soltanto una cosa brutta; 3) tutto sommato, fosse una buona cosa». Per l'efficacia percepita: «Con quale delle seguenti frasi si trova più d'accordo? 1) La nostra democrazia funziona bene; 2) La nostra democrazia ha molti difetti, ma essa funziona; 3) La nostra democrazia sta peggiorando, e tra poco non funzionerà affatto».

${ }^{8}$ Se si escludono $\mathrm{i}$ «non so» e le risposte mancanti, il favore per la democrazia appare ancora più forte: la legittimità diffusa cresce al $79 \%$ in Portogallo, al $78 \%$ in Spagna, al $75 \%$ in Italia e all' $89 \%$ in Grecia; l'efficacia percepita sale all' $86 \%$ in Portogallo, al $77 \%$ in Spagna, al $69 \%$ in Italia e all' $85 \%$ in Grecia. 
mità percepita. In Portogallo è più elevato il numero di «non risposte» (una caratteristica che contraddistingue questo paese anche sulle altre dimensioni rilevate). L'efficacia percepita in Portogallo e Italia è inferiore che in Spagna e Grecia.

Per quanto riguarda il passato autoritario, i greci sono più critici degli intervistati portoghesi, spagnoli e italiani, che invece esprimono un certo grado di ambiguità e di ambivalenza. Oltre a questi pareri misti, in Portogallo e Spagna vi sono percentuali più alte $(13 \%$ e $17 \%$, rispettivamente) di giudizi positivi sul passato. Tuttavia, percentuali così elevate non sono sorprendenti se si considerano la durata del salazarismo (quasi mezzo secolo) e del franchismo (quasi quarant'anni) e l'inevitabile attaccamento al regime cui danno luogo periodi così lunghi, specie tra le generazioni più anziane.

Un confronto tra le risposte «filo-autoritarie» ai primi due quesiti mette in evidenza un'interessante regolarità. In Italia il $6 \%$ valuta favorevolmente il vecchio regime autoritario, mentre il $13 \%$ afferma che in astratto «un regime autoritario, una dittatura, è preferibile» in alcuni casi. Se i primi possono essere considerati «vecchi autoritari» e i secondi «neo-autoritari», se ne può inferire che gli atteggiamenti favorevoli all'autoritarismo non sono riconducibili soltanto a un ricordo positivo del regime di Mussolini.

Succede il contrario in Portogallo e Spagna, dove coloro che in astratto preferiscono un regime autoritario $(9 \%$ e $10 \%$, rispettivamente) sono meno numerosi di coloro che ritengono positivi i regimi autoritari del passato (13\% e $17 \%)$. Ciò suggerisce che in Spagna e Portogallo alcuni nostalgici sostenitori del passato si siano convertiti a ideali democratici ${ }^{9}$. Questi risultati sono conformi a quelli di altre ricerche, secondo cui la maggior parte di coloro che nutrivano atteggiamenti favorevoli verso Franco e si identificavano ideologicamente con il franchismo riconoscono la legittimità del nuovo regime: solo il $4 \%$ delle per-

${ }^{9}$ In Portogallo, Spagna e Italia coloro che Morlino e Mattei chiamano «neodemocratici» (cioè coloro che accettano la legittimità democratica ma allo stesso tempo esprimono un parere favorevole sul passato) rappresentano il gruppo più grande, incidendo, rispettivamente, per il $46 \%$, il $47 \%$ e il $30 \%$. I «neo-autoritari», i quali preferiscono un regime autoritario ma giudicano negativamente le esperienze del passato, sono relativamente più numerosi degli «autoritari», coloro che preferiscono un regime autoritario e giudicano favorevolmente il passato: $10 \%$ contro $6 \%$ in Portogallo; $4 \%$ contro $3 \%$ in Grecia; $12 \%$ conto $4 \%$ in Italia; ma $7 \%$ contro $9 \%$ in Spagna (Morlino e Mattei 1992 , 142-143). 
sone intervistate dall'agenzia Data nel 1981 si identificava totalmente nel franchismo e esprimeva posizioni chiaramente antidemocratiche (vedi Linz et al. 1981, 614).

La conclusione generale da trarre da questi dati è che nel 1985 nell'Europa del Sud non vi sono alternative agli attuali ordinamenti democratici: definitivamente tramontata la storia recente (con la sua serie di dittatori, interventi militari nella politica e sistemi monopartitici), né gli «autoritari» né i «neo-autoritari» pongono serie sfide al regime democratico ${ }^{10}$.

Un altro modo di considerare la legittimità democratica suggerito da Lipset e leggermente modificato da Linz e da altri studiosi del caso spagnolo - è ignorare la dimensione inerente al passato (nonché l'esistenza di alternative percepite) e considerare unicamente le due dimensioni principali riguardanti la legittimità diffusa e l'efficacia percepita (Lipset 1959; GómezReino 1980; Linz et al. 1981). Con queste due dimensioni possiamo elaborare una tipologia che prevede i seguenti tipi: «democratici convinti», «soddisfatti», «critici» e «antidemocratici» ${ }^{11}$. I dati riportati nella fig. 1 evidenziano in modo ancora più netto come siano pochi gli europei del Sud a nutrire atteggiamenti sfavorevoli verso gli attuali regimi democratici. Gli antidemocratici, che esprimono valutazioni negative sia verso la legittimità diffusa sia verso l'efficacia percepita, rappresentano una piccola minoranza in tutti e quattro i paesi. All'estremo opposto, in Portogallo, Spagna e Grecia, tre persone su quattro possono essere definite democratici convinti, così come due italiani su tre.

Tutti e quattro i regimi democratici godono di un elevato livello di legittimità, ma l'Italia presenta una configurazione particolare, con un numero sensibilmente più elevato di critici, cui corrisponde una più ridotta presenza di democratici convinti. Se negli altri tre paesi il rapporto fra coloro che appoggiano senza riserve la democrazia e i «critici» varia da 8:1 (Grecia e Portogallo) a 6:1 (Spagna), in Italia tale rapporto scende a poco

${ }^{10}$ Questa conclusione è rafforzata se si tiene conto dell'età di coloro che nutrono atteggiamenti autoritari (vedi Morlino e Mattei 1992; Montero e Torcal 1990; Heimer et al. 1989).

11 I «democratici convinti» preferiscono il sistema democratico e ritengono che la democrazia funzioni bene, anche se presenta alcuni difetti. All'altro estremo ci sono gli «anti-democratici». I «soddisfatti» esprimono una preferenza per un regime autoritario, ma ritengono che la democrazia funzioni; mentre $\mathrm{i}$ «critici» valutano positivamente la legittimità diffusa e negativamente l'efficacia percepita. 


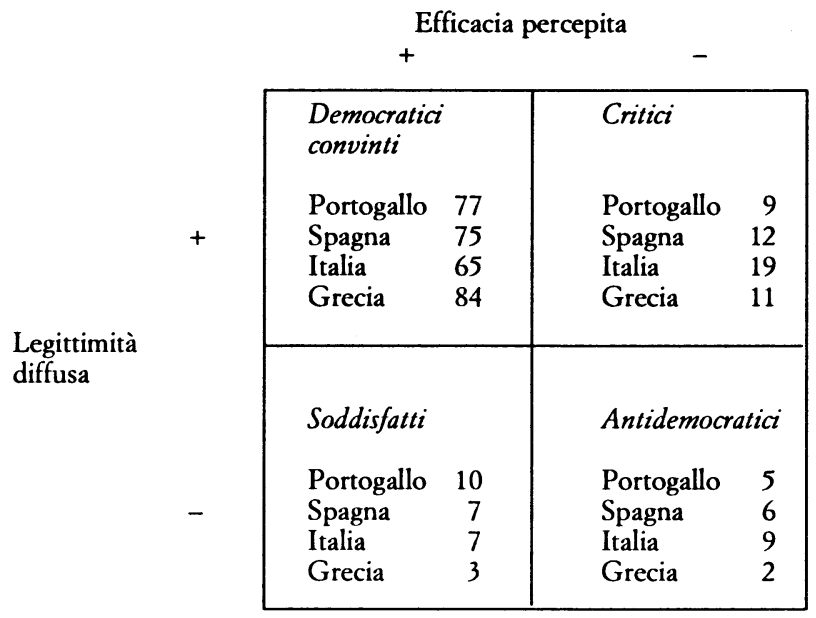

Fig. 1. Legittimità democratica ed efficacia percepita nell'Europa del Sud (valori percentuali per paese)

più di 3:1. Ciò che contraddistingue, infatti, l'Italia è un minor livello di efficacia percepita: il $28 \%$ degli intervistati ritiene che la democrazia «sta peggiorando e non funziona».

Questa conclusione è congruente con i risultati di molte altre ricerche, come si evince dai dati dell'Eurobarometro riportati nella tab. 2. Mentre la soddisfazione per il funzionamento della democrazia in Spagna e Portogallo è prossima alla media Cee (e la Grecia presenta un livello solo un poco inferiore), l'Italia risulta assai distanziata, con un livello minimo di soddisfazione per il funzionamento della democrazia ${ }^{12}$.

Anche i due principali indicatori comportamentali di legittimità - l'ordine civile e i partiti pro-regime - testimoniano dei livelli relativamente elevati di favore per la democrazia in tutti e quattro i paesi a metà degli anni ottanta - un periodo di forte sviluppo economico e in cui il regime di ognuno di questi paesi era pienamente consolidato. Il problema del terrorismo e della violenza civile, ancora presente in Italia, Spagna e Grecia all'inizio degli anni ottanta, si stava gradualmente esaurendo, anche se con ritmi diversi. In Spagna gli atteggiamenti antidemocratici

12 Nel sondaggio Eurobarometro veniva posto il seguente quesito: «Lei quanto è soddisfatto del modo in cui funziona la democrazia?» II testo somiglia a quello usato nel Four Nation Survey e comunica l'idea dell'efficacia percepita (vedi Schmitt 1983). Per i bassi valori riscontrati in Italia, vedi Sani (1980); Guidorossi e Sani (1985). 
ТАв. 2. La soddisfazione per la democrazia, $1985-1990$ (percentuale di intervistati che si dichiarano «molto» $o$ «abbastanza» soddisfatti)

\begin{tabular}{lcccc}
\hline & 1985 & 1987 & 1989 & 1990 \\
\hline Portogallo & 34 & 70 & 60 & 71 \\
Spagna & 51 & 55 & 60 & 57 \\
Italia & 28 & 26 & 27 & 21 \\
Grecia & 51 & 49 & 52 & 44 \\
Comunità europea & 58 & 57 & 66 & 62 \\
\hline
\end{tabular}

Fonte: Eurobarometro, vari anni.

erano praticamente assenti a livello nazionale; erano riscontrabili posizioni estreme soltanto riguardo al problema regionale ${ }^{13}$. In Italia stavano scomparendo le posizioni estreme in seguito alla crisi della destra (specie del Msi), alla profonda trasformazione del partito comunista a partire dai primi anni settanta, al suo ruolo decisivo nel sostenere la democrazia durante la fase più dura del terrorismo alla fine degli anni settanta, nonché al suo appoggio «esterno» ai governi del periodo 1976-79. Potevano considerarsi antiregime (piuttosto che antidemocratiche) soltanto alcune sparute minoranze, collocate agli estremi dello spettro politico. In Grecia e Portogallo godevano di maggiori consensi i partiti antiregime (specie quelli della sinistra comunista, che ricevettero, rispettivamente, il $12 \%$ e il $15 \%$ del voto nelle precedenti elezioni), ma anch'essi tendevano al declino ${ }^{14}$.

A questo punto va esplicitato un ulteriore problema. Dalla tab. 1 e dalla fig. 1 è evidente che l'Italia e la Spagna esprimono le più alte percentuali di antidemocratici; che questa tendenza è ulteriormente confermata per l'illegittimità; che l'Italia, seguita dalla Spagna, possiede la più alta percentuale di critici. L'Italia, inoltre, presenta la più bassa percentuale di democratici convinti, e in Spagna si registrano le valutazioni più favorevoli del passato franchista. Allo stesso tempo, in questi due paesi si hanno piccoli gruppi estremi e partiti comunisti che sono pienamente integrati nel regime e che lo appoggiano. Portogallo e Grecia, che presentano $\mathrm{i}$ livelli più bassi di illegittimità e il minor nu-

13 Pensiamo al caso di Herri Batasuna, nonché alla dinamica del sistema regionale dei partiti polarizzato e frammentato nella regione basca (vedi Linz e Montero 1986; Llera 1989).

${ }^{14}$ Questa è soltanto un'analisi molto sommaria di due fenomeni molto diversi tra loro ma salienti. 
TAB. 3. Legittimità democratica ed efficacia percepita per autocollocazione sinistra-destra (percentuali di riga)

\begin{tabular}{|c|c|c|c|c|c|c|}
\hline & $\begin{array}{l}\text { Democratici } \\
\text { convinti }\end{array}$ & Critici & $\begin{array}{l}\text { Soddi- } \\
\text { sfatti }\end{array}$ & $\begin{array}{c}\text { Anti- } \\
\text { democratici }\end{array}$ & Totale & $(\mathrm{N})$ \\
\hline \multicolumn{7}{|l|}{ Portogallo } \\
\hline $\begin{array}{l}\text { Sinistra } \\
\text { Centro-sinistra } \\
\text { Centro } \\
\text { Centro-destra } \\
\text { Destra }\end{array}$ & $\begin{array}{l}75 \\
79 \\
81 \\
71 \\
63\end{array}$ & $\begin{array}{r}9 \\
12 \\
7 \\
8 \\
12\end{array}$ & $\begin{array}{r}12 \\
7 \\
7 \\
15 \\
33\end{array}$ & $\begin{array}{r}5 \\
2 \\
4 \\
8 \\
12\end{array}$ & $\begin{array}{r}8 \\
17 \\
40 \\
13 \\
4\end{array}$ & $\begin{array}{r}(107) \\
(225) \\
(520) \\
(171) \\
(57)\end{array}$ \\
\hline Totale & 77 & 9 & 10 & 4 & & (1305) \\
\hline \multicolumn{7}{|l|}{ Spagna } \\
\hline $\begin{array}{l}\text { Sinistra } \\
\text { Centro-sinistra } \\
\text { Centro } \\
\text { Centro-destra } \\
\text { Destra }\end{array}$ & $\begin{array}{l}77 \\
86 \\
74 \\
53 \\
44\end{array}$ & $\begin{array}{r}15 \\
9 \\
11 \\
22 \\
18\end{array}$ & $\begin{array}{r}3 \\
4 \\
9 \\
8 \\
16\end{array}$ & $\begin{array}{r}5 \\
1 \\
6 \\
17 \\
22\end{array}$ & $\begin{array}{r}7 \\
35 \\
28 \\
10 \\
2\end{array}$ & $\begin{array}{r}(137) \\
(665) \\
(546) \\
(190) \\
(45)\end{array}$ \\
\hline Totale & 75 & 12 & 7 & 6 & & (1926) \\
\hline \multicolumn{7}{|l|}{ Italia } \\
\hline $\begin{array}{l}\text { Sinistra } \\
\text { Centro-sinistra } \\
\text { Centro } \\
\text { Centro-destra } \\
\text { Destra }\end{array}$ & $\begin{array}{l}53 \\
64 \\
76 \\
60 \\
51\end{array}$ & $\begin{array}{l}32 \\
22 \\
26 \\
11 \\
17\end{array}$ & $\begin{array}{r}5 \\
5 \\
8 \\
15 \\
9\end{array}$ & $\begin{array}{r}10 \\
8 \\
4 \\
14 \\
23\end{array}$ & $\begin{array}{r}17 \\
18 \\
29 \\
8 \\
6\end{array}$ & $\begin{array}{r}(285) \\
(307) \\
(566) \\
(128) \\
(96)\end{array}$ \\
\hline Totale & 65 & 19 & 7 & 9 & & $(1680)$ \\
\hline \multicolumn{7}{|l|}{ Grecia } \\
\hline $\begin{array}{l}\text { Sinistra } \\
\text { Centro-sinistra } \\
\text { Centro } \\
\text { Centro-destra } \\
\text { Destra }\end{array}$ & $\begin{array}{l}89 \\
95 \\
87 \\
67 \\
54\end{array}$ & $\begin{array}{r}9 \\
3 \\
9 \\
20 \\
27\end{array}$ & $\begin{array}{l}2 \\
2 \\
3 \\
6 \\
7\end{array}$ & $\begin{array}{r}- \\
- \\
1 \\
6 \\
12\end{array}$ & $\begin{array}{r}17 \\
23 \\
34 \\
9 \\
10\end{array}$ & $\begin{array}{l}(306) \\
(419) \\
(612) \\
(160) \\
(182)\end{array}$ \\
\hline Totale & 84 & 11 & 3 & 2 & & (1795) \\
\hline
\end{tabular}

mero di antidemocratici, avevano, a metà degli anni ottanta, partiti comunisti più estremisti di quelli degli altri due paesi. Come possiamo dar conto di questa contraddizione?

Questi risultati rispecchiano innanzitutto il noto divario tra atteggiamenti e comportamenti, nonché lo specifico divario tra 
gli atteggiamenti presenti nella società e la misura in cui essi vengono «tradotti politicamente» mediante azioni di gruppi e leader. Ma vi è anche una seconda importante spiegazione di questa contraddizione, che emerge dalla tabulazione incrociata dei quattro tipi della fig. 1 e l'autocollocazione sinistra-destra degli intervistati (tab. 3). Gli intervistati di sinistra manifestano atteggiamenti più favorevoli di quelli di destra verso la democrazia. Se si considera che il sondaggio è stato condotto negli anni ottanta, questo risultato induce a contestare l'assunto ricorrente circa la natura antidemocratica della sinistra. Questa considerazione permette anche di considerare la salienza dell'ideologia nello spiegare il grado e le caratteristiche della legittimità democratica.

Volgiamo ora l'attenzione a due risultati insoliti riguardanti il Portogallo e la Grecia. In Portogallo vi è un'incidenza particolarmente elevata di «non risposte». In molte tabelle, sia in questo articolo sia in altre ricerche pubblicate (vedi, ad esempio, Bacalhau 1978), essa è il doppio dei tassi di non-risposta negli altri tre paesi dell'Europa del Sud. Senza ricorrere a una trattazione approfondita del significato e dei problemi metodologici implicati da questo genere di risposte nei sondaggi (Passeron 1982,83$)$, riteniamo che in questo caso un tasso così elevato di «non-risposte» non vada interpretato come indizio di mancato sostegno per il regime democratico.

Una prima interpretazione di questo risultato rinvia ad alcune caratteristiche del contesto portoghese. Rispetto agli altri tre paesi, a metà degli anni ottanta il Portogallo era caratterizzato da un minor grado di modernizzazione in termini di istruzione, urbanizzazione, struttura della forza lavoro e sviluppo dei mezzi di comunicazione. Si tratta di fattori che favoriscono bassi tassi di risposta nei sondaggi di opinione.

Secondo un'altra possibile interpretazione, le mancate risposte sono da mettere in relazione con alcuni aspetti congiunturali della politica portoghese. Il sondaggio è stato realizzato in un periodo di trasformazione politica per il Portogallo: l'incertezza era così diffusa che essa può essersi estesa fino agli atteggiamenti verso la democrazia. Va ricordato che nelle elezioni del 1985 vi era un partito nuovo (il Partito di rinnovamento democratico del presidente António Ramalho Eanes) che raccolse ben il $18 \%$ del voti espressi. L'instabilità elettorale di quelle elezioni è stata la più alta dalla fondazione della democrazia portoghese. Nel corso dei due anni successivi, inoltre, il Partito 
socialdemocratico (Psd) è diventato l'attore dominante dell'arena politica (vedi Bruneau e McLeod 1986).

Dato che il Portogallo è il paese dell'Europa occidentale còn la più lunga esperienza autoritaria, praticamente nessuno dei suoi cittadini aveva un'esperienza democratica di qualsivoglia genere. In questo periodo la cultura politica portoghese era caratterizzata dal cinismo e dall'ignoranza politica (Bruneau 1985). L'instabilità del governo e le difficoltà economiche del periodo immediatamente antecedente al sondaggio del 1985 hanno influenzato in modo diretto e significativo la soddisfazione per la democrazia e forse provocato una maggior incertezza negli orientamenti democratici di fondo. Alcuni primi indizi che confermano questa ipotesi di «incertezza autentica» sono riscontrabili nella tab. 2: i livelli di soddisfazione per la democrazia portoghese erano davvero bassi nel 1985. Dopo due anni, tuttavia, essi erano ben al di sopra della media Cee. Più avanti torneremo a occuparci di questa ipotesi di «autentica incertezza».

La seconda anomalia degna di nota riguarda l'elevata percentuale di atteggiamenti favorevoli su ciascuna dimensione della legittimità (tab. 1) e l'alta percentuale di democratici convinti (fig. 1) in Grecia. Le cifre sono così alte da suscitare qualche sospetto in merito alla rappresentatività del campione ${ }^{15}$. Nonostante queste riflessioni sul campionamento, per vari aspetti i dati descrivono in modo adeguato la cultura politica greca. In primo luogo, le interviste sono state effettuate durante la fase più intensa della campagna elettorale di quell'anno, quando probabilmente erano al culmine le dichiarazioni pubbliche di sostegno alla democrazia. In secondo luogo, i dati dell'Eurobarometro tendono a corroborare questi risultati. Come si evince dalla tab. 2, la soddisfazione per la democrazia nel 1985 era molto più alta in Grecia che in Italia e Portogallo. In terzo luogo, diversi studiosi, tra cui Diamandouros (1983), Dimitras (1987; 1990), Kafetsis (1988) e Papacosma (1988), hanno definito la cultura politica greca come altamente politicizzata, con elevati livelli di impegno politico popolare, intensi convinci-

15 In un caso la procedura di campionamento può avere inficiato la rappresentatività del campione: per ridurre $i$ costi, la quota di intervistati residenti nei comuni più piccoli è stata estratta in zone prossime ai grandi centri urbani. Di conseguenza, sono sottorappresentati $i$ greci che abitano in zone rurali isolate, $i$ quali probabilmente si interessano meno di politica. 
menti politici, grandi gruppi radicalizzati (specie tra i giovani) e atteggiamenti favorevoli verso la politica in generale.

Queste due anomalie relative al Portogallo e alla Grecia fanno pensare che lo strumento analitico adottato sia molto sensibile a variazioni di atteggiamenti di massa provocati da specifici eventi politici o da particolari contingenze ${ }^{16}$. Tuttavia, la rilevazione di queste tendenze richiede disegni di ricerca basati sui panels oppure indagini ripetute nel tempo, come i sondaggi Eurobarometro. La maggior parte della nostra analisi è basata su un unico sondaggio in ciascun paese, per cui non dedicheremo molto spazio all'esame delle variazioni degli atteggiamenti nel tempo.

La principale conclusione da trarre da questi dati è che a metà degli anni ottanta il processo di consolidamento democratico era per lo più compiuto. Nei periodi precedenti i livelli di soddisfazione e di efficacia percepita erano molto inferiori, specie se si considerano i relativi contesti politici, caratterizzati da aspettative crescenti e dalla crisi economica, che avevano diffuso frustrazione, disillusione e disincanto (Maravall 1991). Tuttavia, le percezioni negative dell'efficacia democratica non hanno influito nella stessa misura sul livello di legittimità; ciò in conformità con quanto sostiene Linz, secondo cui la legittimità democratica è relativamente autonoma dall'efficacia percepita. Anche se questa autonomia è minore nelle prime fasi di cambiamento di regime, essa ha aiutato le nuove democrazie dell'Europa del Sud ad affrontare i problemi dovuti al divario tra aspettative sociali e difficoltà e a risolvere rilevanti problemi economici e sociali (Linz 1990; Linz e Stepan 1989).

Come mettono in evidenza i dati della tab. 4, le correlazioni tra preferenza per la democrazia rispetto all'autoritarismo (legittimità diffusa), da una parte, e soddisfazione per il funzionamento della democrazia (efficacia percepita), dall'altra, sono piuttosto basse. I coefficienti di correlazione non raggiungono mai il valore di 0,35 . Così in base a un grezzo indicatore stati-

${ }^{16} \grave{E}$ interessante prendere in esame le possibili relazioni tra i nostri dati sulla soddisfazione per la democrazia con alcuni eventi politici. Ad esempio, nella tab. 3 vi è un contrasto tra i bassi livelli di continuità in Italia e quelli elevati in Spagna, i quali coincidono con il governo maggioritario socialista, insediato sin dal 1982. Allo stesso tempo, l'andamento negativo in Grecia si sovrappone alle difficoltà del Pasok e a un'alternanza al governo con Nea Democratia in seguito a tre tornate elettorali succedutesi in un breve arco di tempo. L'aumento in Portogallo si sviluppa parallelamente alla formazione del primo governo maggioritario omogeneo da parte dei socialdemocratici. 
ТАв. 4. Coefficienti di correlazione tra legittimità diffusa, efficacia percepita e opinione sul passato autoritario

\begin{tabular}{lcc}
\hline & $\begin{array}{c}\text { La democrazia } \\
\text { funziona }\end{array}$ & $\begin{array}{c}\text { Opinione negativa sul } \\
\text { passato autoritario }\end{array}$ \\
\hline La democrazia è preferibile & & \\
Portogallo & 0,16 & 0,25 \\
Spagna & 0,29 & 0,33 \\
Italia & 0,24 & 0,26 \\
Grecia & 0,21 & 0,34 \\
La democrazia funziona & & \\
Portogallo & & \\
Spagna & & 0,09 \\
Italia & & 0,19 \\
Grecia & & 0,00 \\
\hline
\end{tabular}

stico, possiamo affermare che l'efficacia «spiega» meno del $10 \%$ della varianza della variabile «legittimità diffusa». I quesiti relativi alla preferenza per un regime piuttosto che per un altro e alla soddisfazione sembrano riferirsi a dimensioni diverse, seppure collegate tra loro sul piano teorico. In linea con la nostra ipotesi, gli europei del Sud sono stati in grado di distinguere tra legittimità ed efficacia, al punto tale che l'insoddisfazione per il funzionamento delle loro democrazie non ha portato a una diminuzione della preferenza generale per un regime democratico ${ }^{17}$.

Un esame più articolato del caso spagnolo rende questa conclusione ancora più convincente. Nei tre sondaggi effettuati da Barnes et al. nel 1978, 1980 e 1984 sono stati usati quesiti relativi alla legittimità e all'efficacia analoghi ai nostri. I loro risultati confermano entrambe le suddette conclusioni. In primo luogo, essi indicano che l'autonomia della legittimità dall'efficacia è aumentata nel tempo, mano a mano che il nuovo regime si consolidava: la correlazione tra gli indicatori di orientamenti fa-

17 La distinzione tra legittimità ed efficacia si manifesta a livello di comportamento di voto. Una prima analisi degli atteggiamenti relativi alla legittimità diffusa e all'efficacia percepita tra diversi gruppi di votanti indica una più estesa legittimità diffusa, senza differenze significative tra essi; anzi, le differenze sono molto più marcate quando si esamina l'efficacia percepita. É evidente che in ogni paese tali differenze ricalcano la linea di demarcazione tra partiti di governo e partiti di opposizione. 
TAB. 5. Andamento degli atteggiamenti verso la legittimità denocratica in Spagna, 1978 1989 (valori percentuali)

\begin{tabular}{lrrrr}
\hline & 1978 & 1980 & 1985 & 1989 \\
\hline Democratici convinti & 74 & 46 & 75 & 76 \\
Critici & 11 & 32 & 12 & 11 \\
Soddisfatti & 3 & 4 & 7 & 6 \\
Antidemocratici & 12 & 18 & 6 & 7 \\
Totale & 100 & 100 & 100 & 100 \\
$(\mathrm{~N})$ & $(1190)$ & $(4784)$ & $(1926)$ & $(2472)$ \\
\hline
\end{tabular}

Fonte: per il 1978 e il 1980, Linz et al. (1981, 628); per il 1985 e il 1989, Centro de Investigaciones Sociológicas, Madrid.

vorevoli all'ordinamento democratico e quelli di soddisfazione per «come la democrazia sta funzionando in Spagna» era pari a 0,81 nel 1978, 0,68 nel 1980 e 0,57 nel 1984 (McDonough et al. $1986,751)$. In secondo luogo, nonostante l'accumularsi nel periodo 1980-81 di problemi irrisolti (tra cui la ripresa del terrorismo basco; aspri scontri politici sull'autonomia regionale; la profonda crisi del partito di governo, l'Ucd; e persino un tentato golpe nel febbraio 1981), la legittimità di fondo della nuova democrazia spagnola è rimasta in gran parte salda. Nella tab. 5 si vede che il numero di «critici» (leali democratici, che sono però insoddisfatti delle prestazioni delle istituzioni) si è triplicato, e che vi è stata una diminuzione di questi giudizi negativi soltanto dopo l'avvento al potere del Psoe nel 1982. Tuttavia, la legittimità di fondo della democrazia non è stata erosa dal disagio economico né dal pessimismo politico; e il numero di intervistati con sentimenti antidemocratici è rimasto decisamente basso.

Insomma, gli spagnoli sono stati capaci di distinguere tra rendimento istituzionale e regime democratico, e non hanno attribuito i loro problemi alla democrazia. Anche se l'aumento del numero di critici alla fine ha prodotto un profondo riallineamento elettorale, esso non è stato accompagnato da un aumento significativo del consenso per soluzioni antidemocratiche (Linz 1989; Montero e Torcal 1990; Maravall e Santamaria 1986; Di Palma 1984). Questo quadro nel 1985 risulta valido anche per l'Italia, per quanto essa sia caratterizzata da un grado assai inferiore di soddisfazione per il funzionamento della democrazia. 
Questa analisi preliminare dei dati del sondaggio suggerisce due importanti conclusioni generali. La prima è che il livello di legittimità è elevato in tutti e quattro i paesi (specie in Grecia), anche se vi sono alcune anomalie relative al Portogallo. In secondo luogo, il rapporto tra legittimità del regime e percezione dell'efficacia del governo è piuttosto debole. Questo rapporto è debole a prescindere dalle sostanziali differenze nel grado di efficacia percepita da paese a paese: la percezione di efficacia era bassa in Spagna e Portogallo all'inizio degli anni ottanta, ma da allora è aumentata in modo significativo; le tendenze sono invece diverse in Italia (dove i livelli sono rimasti sempre assai bassi) e in Grecia (dove l'efficacia percepita è un po' diminuita nel 1990). In nessun caso, tuttavia, vi è stata una diminuzione del grado di legittimità diffusa (per dati sulla Spagna, vedi Montero 1992; per il Portogallo, Bruneau 1983).

\section{Alcune spiegazioni}

Occorre ora cercare di spiegare perché questi nuovi regimi hanno conseguito un elevato livello di legittimità. È importante, inoltre, approfondire comunque il significato di legittimità. In questo modo, esploreremo anche la realtà che sta dietro ai risultati del sondaggio ricordati in precedenza.

La prima, più evidente spiegazione degli elevati livelli di legittimità riconosciuti a questi regimi democratici riguarda la mancanza di chiare alternative alla democrazia: in ognuno dei quattro paesi vi è stata (in misura diversa) una fondamentale delegittimazione dei regimi autoritari del passato. Un punto da sottolineare è che i sentimenti di sostegno diffuso sono equidistribuiti fra tutti i settori della società, a parte qualche eccezione di rilievo ${ }^{18}$. Un'analisi approfondita dei possibili rapporti tra

18 Ad esempio, costituisce una parziale eccezione la correlazione negativa ( $r=-$ $0,30)$ tra pratica religiosa e legittimità democratica in Spagna. Lo stesso rapporto è molto più debole negli altri paesi: $-0,16$ in Grecia; $-0,10$ in Portogallo; 0,07 in Italia. La salienza politica di questa frattura è stata oggetto di molti studi: Gunther et al. (1986); Linz et al. (1981); Montero (1986); Pérez Diaz (1987). Un'altra eccezione interessante è la regione basca in Spagna, che in questo saggio non viene considerata. Per i risultati di sondaggi riguardanti la legittimità accordata alla monarchia costituzionale spagnola nella regione basca, si rinvia a Llera (1992) e Shabad (1993). Altre relazioni interessanti tra età e reddito, da una parte, e un «indice di alienazione politica», dall'altra, verranno commentate nel prosieguo di questo articolo. 
legittimità diffusa, legittimità per difetto di alternative ed efficacia percepita, da una parte, e le variabili socio-economiche che di solito vengono inserite nei sondaggi (come reddito, status occupazionale, classe sociale, livello di istruzione, età e pratica religiosa), dall'altra, danno luogo a correlazioni (anche molto) basse. In altri termini, non vi è alcun particolare gruppo sociale o demografico che si differenzi dal resto della società per un livello più alto o più basso di favore per la democrazia ${ }^{19}$.

Le correlazioni con un'importante variabile politica, l'autocollocazione sul continuum sinistra-destra, sono invece ben più consistenti. Abbiamo già osservato come questa variabile sia significativamente collegata a misure indipendenti del favore per la democrazia. Al fine di estendere ulteriormente quell'analisi, abbiamo costruito un «indice di atteggiamenti favorevoli alla democrazia» mediante la combinazione delle risposte degli intervistati alle tre domande principali di questa ricerca. Si è giunti così ad avere tre categorie di intervistati: una categoria di «forte sostegno» che comprende coloro che hanno reagito nel modo più favorevole ai quesiti sulla legittimità diffusa e sull'efficacia percepita e nel modo più sfavorevole verso il passato autoritario; una categoria di «sostegno nullo» che comprende coloro che hanno reagito in modo negativo o indifferente ai quesiti sulla legittimità diffusa e sull'efficacia percepita e in modo favorevole o misto verso il passato autoritario; e una categoria di «sostegno debole» che raccoglie coloro che hanno espresso risposte miste o intermedie $e^{20}$. Il rapporto tra questo indice e l'autocollocazione sinistra-destra presenta variazioni significative da paese a paese. Esso è più forte in Spagna e Grecia, dove si hanno correlazioni di 0,45 e 0,47 , rispettivamente; mentre in Italia e Portogallo i valori corrispondenti sono, rispettivamente, 0,16 e 0,23 .

La fig. 2 rappresenta l'incrocio tra l'autocollocazione sinistra-destra e l'indice di atteggiamenti favorevoli verso la democrazia (la categoria intermedia di «sostegno debole» è stata

${ }^{19} \mathrm{McDonough}$ et al. (1986) arrivano a conclusioni analoghe in una ricerca su un panel spagnolo dal 1978 al 1984.

${ }^{20}$ Le risposte «non so» e quelle mancanti sono state escluse dai calcoli. Gli atteggiamenti di favore per la democrazia sono più diffusi in Grecia, dove il $56 \%$ dei soggetti ricadevano nella categoria del «sostegno forte» (contro il $32 \%$ in Portogallo, il $28 \%$ in Spagna e il $27 \%$ in Italia). La categoria «sostegno nullo» dà luogo a una distribuzione con caratteristiche opposte, e raccoglie il $26 \%$ degli spagnoli e degli italiani, il $19 \%$ dei portoghesi e il $13 \%$ dei greci. 

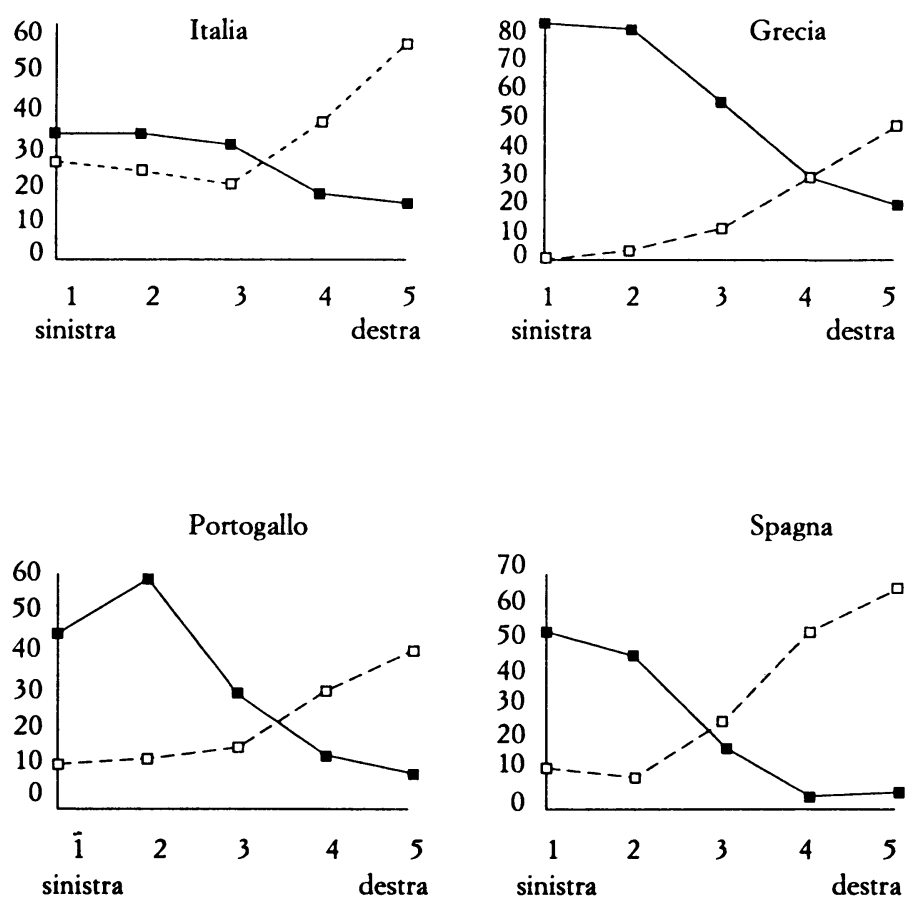

Legenda: - forte $\square$ nessuna legittimità

FIG. 2. Legittimità democratica per autocollocazione sinistra-destra (valori percentuali)

esclusa per motivi di chiarezza). La forza di questa relazione in ciascun paese è evidente. La rappresentazione grafica, tra l'altro, permette di evitare un errore interpretativo in cui sarebbe stato possibile incorrere esaminando soltanto i coefficienti di correlazione: in Portogallo la correlazione tra la variabile sinistra-destra e l'indice di sostegno si attenua a causa di una relazione curvilinea.

Le specifiche correlazioni tra autocollocazione sinistra-destra e singole componenti dell'indice mettono in evidenza alcuni patterns che aiutano ad interpretare questi risultati. La relazione tra valutazione del passato autoritario e autocollocazione sinistra-destra dimostra che vi è una maggiore polarizzazione di queste opinioni lungo la dimensione sinistra-destra in Spagna (il coefficiente di correlazione assume il valore di 0,53$)$ che in Grecia $(0,46)$, Portogallo $(0,33)$ e Italia $(0,31)$. Anche per quanto ri- 
guarda la relazione tra percezione dell'efficacia governativa e autocollocazione sinistra-destra, $i$ coefficienti sono più bassi in Italia $(0,11)$ e Portogallo $(0,05)$ che in Spagna $(0,22)$ e Grecia $(0,26)$.

Come vanno interpretati questi risultati? Secondo le teorie sulla dimensione sinistra-destra, quest'ultima comprende sia una componente ideologica, sia una di preferenza partitica, il che fa pensare che questi risultati dipendano in parte dagli orientamenti verso $i$ partiti ${ }^{21}$. Di conseguenza, le prestazioni dei partiti al governo (che evidentemente sono salienti per la dimensione dell'efficacia percepita) e le posizioni espresse dai partiti e dai loro leader in merito alla legittimità diffusa esercitano una grande influenza sugli atteggiamenti degli intervistati, che abbiano un partito preferito. Se si introducono le preferenze partitiche come variabile di controllo (vedi tab. 6), questa interpretazione della varianza nel sostegno al sistema consegue una maggiore credibilità. $\mathrm{Vi}$ è una relazione particolarmente forte tra preferenza partitica ed efficacia percepita.

Questo risultato non è inatteso, in quanto, in fin dei conti, si chiede agli intervistati di giudicare l'efficacia di un governo democratico controllato da uno specifico partito o da una particolare coalizione: ad esempio nel 1985, con il Psoe al potere, gli elettori del Psoe giudicarono in modo più favorevole le prestazioni del regime democratico di quanto abbiano fatto i sostenitori di altri partiti; gli elettori del Pasok valutarono la democrazia greca anche più positivamente durante la presenza del $\mathrm{Pa}$ sok al governo; e in Italia i sostenitori dei partiti presenti nel governo di coalizione del 1985 (Psi, Psdi, Pri, Pli e Dc) ritennero migliore il rendimento democratico del regime dei sostenitori dei partiti di opposizione. L'unica eccezione di rilievo riguarda gli elettori socialisti in Portogallo, il cui partito preferito faceva parte di una coalizione governativa in procinto di cadere e si accingeva ad affrontare un lungo periodo all'opposizione.

$\mathrm{Da}$ altri dati riportati nella tab. 6 emerge chiaramente che fattori diversi dalla preferenza partitica contribuiscono a determinare questi patterns. In primo luogo, in ogni paese gli elettori dei partiti di destra hanno dato le risposte meno favorevoli ai quesiti sulla legittimità diffusa e sull'efficacia percepita e le più

${ }^{21}$ Questa è una delle conclusieni principali di Inglehart e Klingemann (1976) e di Arian e Shamir (1983). Si vedano anche Laponce (1981); Sani (1974); Sani e Montero (1986); Klingemann (1987). 
TAB. 6. Legittimità democratica, opinione sul passato autoritario ed efficacia percepita secondo il partito votato (valori percentuali)

\begin{tabular}{|c|c|c|c|c|c|c|c|c|}
\hline Portogallo & Pcp & Ps & Psd & Cds & Altri & & & \\
\hline Democrazia preferibile & 77 & 67 & 64 & 56 & 75 & & & \\
\hline Passato cattivo & 64 & 32 & 17 & 8 & 44 & & & \\
\hline Democrazia funziona $^{a}$ & 82 & 75 & 80 & 65 & 75 & & & \\
\hline Spagna & Pce & Psoe & Cds & Ap & Altri & & & \\
\hline Democrazia preferibile & 89 & 86 & 68 & 53 & 80 & & & \\
\hline Passato cattivo & 71 & 42 & 12 & 1 & 42 & & & \\
\hline Democrazia funziona $^{a}$ & 73 & 84 & 67 & 53 & 74 & & & \\
\hline Italia & Pci & Psi & Psdi & Pri & Dc & Pli & Msi & Altri \\
\hline Democrazia preferibile & 70 & 77 & 88 & 71 & 80 & 67 & 30 & 69 \\
\hline Passato cattivo & 61 & 36 & 42 & 36 & 32 & 29 & 3 & 47 \\
\hline Democrazia funziona $^{a}$ & 50 & 71 & 73 & 84 & 83 & 62 & 39 & 62 \\
\hline Grecia & Kke & Pasok & $\mathrm{Nd}$ & Altri & & & & \\
\hline Democrazia preferibile & 96 & 93 & 75 & 87 & & & & \\
\hline Passato cattivo & 86 & 74 & 26 & 77 & & & & \\
\hline Democrazia funziona ${ }^{a}$ & 86 & 95 & 58 & 78 & & & & \\
\hline
\end{tabular}

- Le percentuali di questa riga sono determinate dall'unione di due risposte favorevoli («la democrazia funziona bene» e «la democrazia ha molti difetti, ma funziona»). Vedi anche tab. 1.

favorevoli sul passato autoritario. In secondo luogo, nonostante i comunisti portoghesi, spagnoli e greci fossero all'opposizione, i loro sostenitori esprimono una maggiore legittimità diffusa della democrazia rispetto ai socialisti, il cui partito era l'unico al governo oppure il partner dominante della coalizione di maggioranza.

In generale, gli intervistati di sinistra mostrano un grado significativamente e sistematicamente maggiore di favore per la democrazia rispetto a quelli di destra. Ciò è particolarmente vero quando si tiene conto di chi sta al governo (ad esempio, aggregando $\mathrm{i}$ «democratici convinti» ai «critici» presentati nella tab. 3). Inoltre, va sottolineato che nel caso spagnolo (per il quale sono disponibili serie storiche di dati) gli intervistati di sinistra erano più favorevoli alla democrazia di quelli di destra persino nel 1978, quando l'Ucd, un partito di centro-destra, era 
al potere. Ancora, in Italia e, in misura minore, in Grecia i partiti sono più salienti e visibili nella società che negli altri due paesi.

Questa conclusione è desumibile da diversi indicatori, ma per ora consideriamone uno soltanto: l'identificazione partiti$\mathrm{Ca}^{22}$. Sono meno numerosi gli spagnoli e i portoghesi che si identificano in un partito (rispettivamente il $43 \%$ e il $48 \%$ ) rispetto agli italiani $(57 \%)$ e ai greci $(72 \%)^{23}$. Benché un'ampia parte della popolazione italiana si identifichi in un partito, la corrispondente relazione tra autocollocazione sinistra-destra e favore per la democrazia è significativamente più bassa che negli altri paesi dell'Europa del Sud. La Spagna, invece, presenta la più bassa quota di identificati e la più forte relazione tra legittimità e dimensione sinistra-destra. Sembra dunque che la componente ideologica del continuum sinistra-destra assuma maggiore importanza se i legami partitici non sono particolarmente diffusi, intensi o salienti. Inoltre, sia le componenti ideologiche che quelle di orientamento verso i partiti sono rilevanti se vi è una forte ed estesa presenza dei partiti nella società, come nel caso di Italia e Grecia. La Spagna, invece, è un caso di debole presenza partitica. Il Portogallo, infine, si colloca in una posizione intermedia.

Insomma, l'autocollocazione sinistra-destra influisce in modo significativo e sistematico sulla propensione dei cittadini a riconoscere la legittimità di un regime e a ritenere efficaci le sue prestazioni. Nei quattro paesi in questione le due componenti di questo fattore sono rilevanti in modi diversi. In Grecia

22 Tra le varie misure che sono state proposte abbiamo scelto quella di Mannheimer e Sani (1987a), i quali ricorrono a due indicatori di preferenza partitica. Il primo è un quesito classico: «Mi dica se si sente molto vicino, vicino, né vicino né lontano, lontano o molto lontano... da ciascuno dei partiti riportati in questo elenco». Il secondo quesito ha una dimensione affettivo-reattiva: «Quando qualcuno critica il partito per il quale lei ha votato alle ultime elezioni, di solito lei come reagisce? 1) mi infastidisce come se stessero criticando me; 2) non mi infastidisce, ma non mi piace; 3) mi è indifferente». Gli intervistati non identificati rappresentano il $57 \%$ degli intervistati spagnoli, il $52 \%$ dei portoghesi, il $43 \%$ degli italiani e il $27 \%$ dei greci. I paesi si ordinano in modo analogo se si considerano unicamente le risposte al quesito «classico»: coloro che si ritengono «molto vicini» o «vicini» a un partito erano il $48 \%$ in Spagna, il $49 \%$ in Portogallo, il $52 \%$ in Italia e il $70 \%$ in Grecia.

${ }^{23}$ Alcuni dati più recenti tratti da un sondaggio Eurobarometro indicano livelli ancora più bassi di identificazione in Spagna, dove il $30 \%$ si dichiara molto vicino, abbastanza vicino o soltanto simpatizzante di un partito; mentre in Grecia gli identificati sono pari al $57 \%$, in Portogallo al $49 \%$, in Italia addirittura al $63 \%$. Si vedano anche Schmitt (1989); Mannheimer e Sani (1987b); Mannheimer (1989); Richardson (1990); Gunther e Montero (1992). 
i due aspetti sembrano rafforzarsi a vicenda. In Portogallo, che presenta una debole relazione tra dimensione sinistra-destra e legittimità democratica, le alternanze al governo sono state più frequenti, la componente ideologica e la polarizzazione più forti. In Italia i partiti sono stati dominanti per un lungo periodo, e la polarizzazione si è molto affievolita nei primi anni ottanta. La Spagna è il paese in cui è più forte la componente ideologica, o meglio la componente delle issues. In tutti questi paesi la dimensione sinistra-destra funge da «strumento di orientamento», una bussola che aiuta a semplificare l'universo politico e a capirlo meglio. Pertanto, l'autocollocazione sinistra-destra possiede un significato e un contenuto autonomi in termini di atteggiamenti politici, compresi quelli connessi alla legittimità; ma questi atteggiamenti non sono necessariamente né direttamente collegati a specifiche fedeltà partitiche ${ }^{24}$.

\section{Atteggiamenti verso la politica}

I dati presentati fino a questo punto indicano che a metà degli anni ottanta i regimi democratici esistenti sono ritenuti legittimi da tutti i settori della società; e non risulta alcun sostegno significativo per alternative non democratiche, per quanto questi atteggiamenti di sostegno siano forse un po' più deboli che negli altri paesi dell'Europa occidentale (specie in relazione all'efficacia percepita).

A questi atteggiamenti verso $i$ regimi politici si accompagnano altri orientamenti generali nei confronti della politica. Nell'ambito del Four Nation Survey, agli intervistati è stato chiesto di scegliere (da un apposito elenco) il termine che meglio descrive i loro sentimenti verso la politica (vedi tab. 7). Un esame paese per paese delle risposte porta alle stesse conclusioni dell'analisi del favore per la democrazia. Gli atteggiamenti più favorevoli nei confronti della politica (interesse, impegno, entusiasmo, passione) si riscontrano in Grecia. Il Portogallo presen-

${ }^{24}$ Vanno senz'altro ulteriormente analizzati $i$ valori e gli atteggiamenti connessi alla variabile sinistra-destra, le cui ambiguità sono già state messe in evidenza da altri studiosi (Van der Eijk e Niemoller 1983). Si vedano anche Sani e Montero (1986) per la Spagna; Sani (1981) per l'Italia e la Spagna; Bacalhau (1978) e Mavrogordatos (1983) per la Grecia; Condomines e Barroso (1984) per Portogallo, Spagna e Grecia. 
TAB. 7. Sentimenti suscitati dalla politica (valori percentuali)

\begin{tabular}{lcccc}
\hline & Portogallo & Spagna & Italia & Grecia \\
\hline Interesse, impegno, & & & & \\
entusiasmo, passione & 20 & 29 & 25 & 65 \\
Indifferenza, diffidenza, noia & 52 & 54 & 47 & 26 \\
Irritazione, disgusto & 16 & 10 & 27 & 8 \\
Non so/nessuna risposta & 12 & 7 & 1 & 1 \\
Totale & 100 & 100 & 100 & 100 \\
\hline
\end{tabular}

ta di nuovo la percentuale più elevata di non-risposte, nonché un elevato numero di risposte che indicano la mancanza di impegno politico. Anche gli spagnoli esprimono un elevato grado di indifferenza rispetto alla politica, ma nel complesso i loro atteggiamenti sono più favorevoli di quelli dei portoghesi. L'Italia si distingue anche in questo caso dagli altri paesi per la forte incidenza di atteggiamenti sfavorevoli verso la politica e di un basso grado di impegno politico. Quasi tre italiani su quattro sono indifferenti oppure negativamente orientati nei confronti della politica.

Questi risultati concordano dunque con quelli commentati in precedenza, in relazione all'efficacia percepita e alla soddisfazione per il funzionamento della democrazia. Quando è stata introdotta l'autocollocazione sinistra-destra in qualità di variabile di controllo, è emerso lo stesso andamento: gli intervistati di sinistra erano più favorevolmente disposti verso la politica, mentre quelli di destra erano negativamente orientati; e gli intervistati di centro e di centro-destra risultavano essere meno impegnati politicamente.

Questi dati suggeriscono che, sotto un profilo importante, l'«eccezionalità dell'Europa del Sud» è molto variegata quanto a cultura politica. Almeno due terzi degli italiani, portoghesi e spagnoli intervistati nell'ambito del Four Nation Survey hanno espresso atteggiamenti indifferenti o negativi verso la politica. Nonostante le nostre conclusioni generali sulla legittimità democratica, questi risultati distinguono i cittadini di questi tre paesi da quelli dell'Europa del Nord.

In quale misura queste valutazioni sono accompagnate $\mathrm{da}$ analoghi orientamenti cognitivi? Questi atteggiamenti si collegano alle percezioni di efficacia personale, cioè ai giudizi degli intervistati circa la propria capacità di influire sulla politica? Al 
ТАВ. 8. Indice di alienazione politica (valori percentuali)

\begin{tabular}{lcccc}
\hline Grado di alienazione & Portogallo & Spagna & Italia & Grecia \\
\hline Forte & 57 & 43 & 57 & 32 \\
Medio & 17 & 19 & 21 & 25 \\
Neutro & 6 & 12 & 9 & 20 \\
Nullo & 3 & 8 & 4 & 15 \\
Non so/nessuna risposta & 16 & 18 & 8 & 7 \\
Totale & 100 & 100 & 100 & 100 \\
\hline
\end{tabular}

fine di affrontare questo argomento abbiamo costruito un «indice di alienazione politica» sulla base delle risposte a tre quesiti riguardanti l'efficacia «interna» ed «esterna»" ti nella tab. 8, mettono in evidenza l'incidenza dell'alienazione e degli atteggiamenti sfavorevoli nei confronti della politica e delle élites politiche, con la consueta eccezione della Grecia.

Questi risultati sono conformi a quelli di diverse altre ricerche. In The Civic Culture, ad esempio, l'Italia della fine degli anni cinquanta veniva caratterizzata come avente una cultura alienata (Almond e Verba 1963). Due decenni più tardi, in un contesto storico alquanto diverso, Sani (1980) chiamava «reticente» la cultura politica italiana. Analogamente, José María Maravall (1984) e, più di recente, Joan Botella (1992), nel descrivere la cultura politica spagnola, hanno parlato di una mancanza di interesse per la politica: Maravall chiama questa combinazione di atteggiamenti favorevoli e sfavorevoli «cinismo democratico»; Botella invece parla di «democraticismo cinico». Maravall sostiene, inoltre, che una visione cinica della politica caratterizza da molto tempo la cultura politica europeo-meridionale, e che questa caratteristica può anche essere considerata un giudizio razionale, fondato su una lunga esperienza della po-

${ }^{25}$ Le tre frasi-stimolo sulle quali si basa il nostro indice sono: 1) «i politici non si preoccupano di gente come me»; 2) «la politica è così complicata che la gente come me non sempre riesce a capire cosa succede»; 3) «quelli che stanno al potere badano sempre ai propri interessi personali». Per un'analisi recente di questi problemi vedi Mattei (1987). La nostra definizione di alienazione politica ricalca quella di Finifter (1970; 1972), specie in relazione alla dimensione dell'impotenza politica. Questa definizione privilegia l'inefficacia, a differenza di altre accezioni, come quella di Gamson (1968), nella quale i politici sono considerati «incompetenti e stupidi», quelle di alienazione «sociale» proposte da diversi autori, il concetto di estrangement proposto da Schwarts $(1973,7)$. Si vedano anche Citrin et al. (1975) e Wright (1970). Sul «cinismo politico» cfr. Fraser (1971) e Agger et al. (1961). 
litica come abuso di potere (1991, 17-18). Entrambi gli studiosi hanno richiamato l'attenzione sull'apparente contraddizione tra atteggiamenti sfavorevoli verso la politica e accettazione di fondo del regime; contraddizione che emerge in modo nitido dai nostri dati.

Di conseguenza, la legittimità dei regimi democratici nell'Europa del Sud può anche essere elevata, ma essa si caratterizza per la presenza di orientamenti affettivi e cognitivi in gran parte sfavorevoli (anche se, ancora una volta, con l'eccezione della Grecia). Pertanto, la legittimità democratica trae forse origine non tanto da un insieme di atteggiamenti favorevoli quanto dall'intensità della reazione contro $i$ regimi autoritari del passato. Ciò aiuta anche a capire l'eccezionalità della Grecia rispetto agli altri tre paesi. Il regime militare greco è durato meno dei regimi autoritari degli altri tre paesi e non si è mai consolidato; la fase violenta e repressiva relativa all'insediamento di quel regime è più recente e quindi più viva nei ricordi dei greci. In linea con ciò i nostri dati attestano che il rifiuto del regime militare da parte della popolazione greca è più esteso ed estremo che negli altri tre paesi dell'Europa del Sud.

\section{Le concezioni di democrazia}

I nostri dati indicano che esiste un ampio favore verso la democrazia nell'Europa del Sud a livello di atteggiamenti e che le alternative non democratiche non raccolgono che pochi consensi in questi quattro paesi. Tuttavia, rimane ancora una domanda cui rispondere: come definiscono la democrazia i cittadini di questi paesi, e quale genere di regime si augurano che si instauri? Il consolidamento democratico non implica solo una mera accettazione del principio astratto di democrazia ma il riconoscimento della legittimità di uno specifico regime. I cittadini non si limitano ad esprimere atteggiamenti verso la democrazia: essi nutrono anche concezioni di democrazia che spesso sono poco strutturate ma più o meno congruenti con i loro orientamenti di fondo. È opportuno esplorare queste concezioni al fine di meglio comprendere le mappe di atteggiamento degli europei del Sud. La ricerca di Putnam (1973) sulle élites politiche britanniche e italiane suggerisce come conoscere le «predisposizioni cognitive» e gli «ideali operativi» degli europei del Sud sia particolarmente importante per capire fino a che punto 
la realtà dei rispettivi paesi si discosta da ciò che la gente ritiene debba essere.

Questa parte della nostra indagine si basa su due diverse (e classiche) domande di sondaggio. Con il primo quesito, che attiene al grado di coinvolgimento personale nei processi decisionali, si chiede agli intervistati di scegliere tra partecipazione diretta alla soluzione dei problemi e accettazione passiva delle decisioni delle autorità ${ }^{26}$. Il secondo propone una scelta tra processi decisionali veloci ed efficienti, da una parte, e processi che prevedono un ricorso esteso a consultazioni e alla partecipazione di istituzioni rappresentative ${ }^{27}$. Incrociando le risposte alle due domande si può sviluppare una tipologia delle concezioni di democrazia, articolata in quattro tipi.

Nella «democrazia decisionista» le autorità del regime sono totalmente responsabili della gestione efficace del paese. In questo tipo di democrazia le strutture di intermediazione occupano una posizione marginale, e la società civile svolge un ruolo passivo nei processi decisionali.

Nella «democrazia rappresentativa», di converso, il ruolo delle strutture di intermediazione è pienamente riconosciuto, ma la responsabilità decisionale appartiene sempre alle autorità. Questa è l'accezione che più si avvicina all'idea di governo rappresentativo, in cui le decisioni vengono prese da rappresentanti eletti, mentre i cittadini svolgono un ruolo relativamente passivo e remissivo.

In una «democrazia populista» non viene accordato alcun ruolo alle associazioni, ai gruppi o ad altre strutture di intermediazione. Le due caratteristiche centrali di questa concezione sono la partecipazione diretta ma non organizzata e l'importanza attribuita all'efficienza dei processi decisionali. L'obiettivo sottostante a questo modello è la massimizzazione dell'efficacia personale e l'identità politica, ma sempre in presenza di una componente autoritaria.

${ }^{26}$ Il testo della domanda era: «Quando nel suo quartiere sorge un problema urgente che la riguarda direttamente, quale tra i seguenti corsi d'azione preferisce? 1) lasciare che decidano le autorità; 2) interpellare le autorità; 3) rivolgersi a gruppi o associazioni; 4) manifestare pubblicamente con altre persone interessate».

27 «Quando le autorità devono risolvere un problema, quale tra i seguenti corsi d'azione dovrebbero seguire? 1) è meglio che prendano decisioni rapide senza consultare i cittadini né le associazioni che li rappresentano; 2) devono sempre consultare i cittadini e le associazioni che li rappresentano, anche se ciò dovesse comportare un ritardo per la soluzione del problema». 
Nella «democrazia partecipativa» la società è attiva nei processi decisionali, nei modi con cui i gruppi e le associazioni formano le loro domande e nelle varie forme di partecipazione diretta quali le manifestazioni pubbliche. In questo modello ci si prefigge di garantire il coinvolgimento della società nel processo decisionale, anche a scapito dell'efficienza.

Lo scopo di questa tipologia è presentare immagini o concetti abbastanza semplici, tali da cogliere le percezioni della gente; non vi è alcuna pretesa di proporre alternative alle teorie più raffinate della democrazia che sono state delineate lungo un percorso di studio molto esteso e articolato (vedi, ad esempio, Sartori 1987).

La fig. 3 riporta, per ogni paese, le percentuali di intervistati che ricadono nelle suddette categorie. Il risultato più clamoroso è che in tutti e quattro i paesi una consistente maggioranza affida i processi decisionali alle autorità. La maggior parte degli europei del Sud esprime una preferenza per un regime che funzioni, senza essere ostacolato da conflitti tra gruppi. Allo stesso tempo anche il ruolo dei cittadini - sotto forma di gruppi e associazioni - viene accettato: ciò che abbiamo etichettato «democrazia rappresentativa» riceve il sostegno di circa la metà della popolazione in ognuno dei quattro paesi. In questo caso viene meno l'eccezionalità greca: $i$ greci scelgono questa opzione più o meno nella stessa misura degli altri europei del Sud.

Se passiamo ad esaminare la seconda preferenza, tuttavia, emergono alcune differenze significative tra i vari paesi. In Portogallo e Italia - entrambi caratterizzati (fino al 1985) da un elevato grado di instabilità governativa - vi è una maggiore preferenza per la democrazia decisionista. In Grecia, invece, si registra un consenso eccezionalmente elevato per la democrazia partecipativa. Degno di nota è poi il caso di Spagna e Italia, dove quasi si bilanciano le preferenze per due concezioni diametralmente opposte: la democrazia decisionista e quella partecipativa. Infine, in tutti e quattro i paesi manca un sostegno di qualche rilievo per la democrazia populista. Ne consegue che in tutta l'Europa del Sud si attribuisce un alto valore al ruolo consultivo delle strutture di intermediazione.

Queste quattro divergenti concezioni di democrazia sono strettamente collegate a più ampi insiemi di preferenze ideologiche e partitiche, così come vengono evidenziate lungo il continuum sinistra-destra. I dati riportati nella tab. 9 mostrano che gli intervistati di sinistra sono quelli più favorevoli alla demo- 


\begin{tabular}{|c|c|c|c|c|}
\hline & $\begin{array}{l}\text { Decisioni } \mathrm{r} \\
\text { senza cons }\end{array}$ & $\begin{array}{l}\text { pide } \\
\text { ltazioni }\end{array}$ & $\begin{array}{l}\text { Consultazio } \\
\text { cittadini e a }\end{array}$ & $\begin{array}{l}\text { e di } \\
\text { sociazioni }\end{array}$ \\
\hline & $\begin{array}{l}\text { Democrazia } \\
\text { decisionista }\end{array}$ & & $\begin{array}{l}\text { Democrazia } \\
\text { rappresentat }\end{array}$ & \\
\hline & $\begin{array}{l}\text { Portogallo } \\
\text { Spagna } \\
\text { Italia } \\
\text { Grecia }\end{array}$ & $\begin{array}{r}36 \\
22 \\
29 \\
7\end{array}$ & $\begin{array}{l}\text { Portogallo } \\
\text { Spagna } \\
\text { Italia } \\
\text { Grecia }\end{array}$ & $\begin{array}{l}51 \\
51 \\
45 \\
53\end{array}$ \\
\hline & $\begin{array}{l}\text { Democrazia } \\
\text { populista }\end{array}$ & & $\begin{array}{l}\text { Democrazia } \\
\text { partecipativa }\end{array}$ & \\
\hline & $\begin{array}{l}\text { Portogallo } \\
\text { Spagna } \\
\text { Italia } \\
\text { Grecia }\end{array}$ & $\begin{array}{l}4 \\
6 \\
8 \\
2\end{array}$ & $\begin{array}{l}\text { Portogallo } \\
\text { Spagna } \\
\text { Italia } \\
\text { Grecia }\end{array}$ & $\begin{array}{l}10 \\
21 \\
18 \\
38\end{array}$ \\
\hline
\end{tabular}

FIG. 3. Concezioni della democrazia nell'Europa del Sud, 1985 (valori percentuali)

crazia partecipativa. La partecipazione popolare, sia in veste consultiva, sia nei processi decisionali, è molto meno caldeggiata dagli intervistati di centro e di destra. La concettualizzazione opposta - la democrazia decisionista - raccoglie i maggiori consensi tra gli intervistati di destra e viene approvata (tranne che in Portogallo) da pochissimi intervistati di sinistra. La democrazia rappresentativa riceve un largo appoggio a prescindere dall'autocollocazione degli intervistati in termini sinistra-destra. Soltanto tra gli intervistati di sinistra in Grecia e Spagna questa non è la forma di democrazia preferita.

Quale ruolo svolgono i partiti politici in queste concettualizzazioni della democrazia? Al fine di indagare sul ruolo preferito da accordare ai partiti, abbiamo costruito un «indice di legittimità partitica» sulla base delle risposte a una serie di quesiti relativi a valutazioni positive $o$ negative dei partiti politici ${ }^{28}$

${ }^{28}$ Questo indice si basa sulle reazioni alle seguenti frasi-stimolo: «I partiti politici dividono la gente»; «I partiti si criticano a vicenda, ma in realtà sono tutti eguali»; «I partiti sono inutili»; «I partiti servono a difendere gli interessi dei vari gruppi e classi sociali»; «Grazie ai partiti la gente può partecipare alla vita politica»; e «Senza partiti non ci può essere democrazia». 
TAB. 9. Concezioni della democrazia secondo l'autocollocazione sinistra-destra (valori percentuali)

\begin{tabular}{|c|c|c|c|c|c|}
\hline & Sinistra & $\begin{array}{l}\text { Centro- } \\
\text { Sinistra }\end{array}$ & Centro & $\begin{array}{c}\text { Centro- } \\
\text { Destra }\end{array}$ & Destra \\
\hline \multicolumn{6}{|c|}{ Democrazia decisionista } \\
\hline $\begin{array}{l}\text { Portogallo } \\
\text { Spagna } \\
\text { Italia } \\
\text { Grecia }\end{array}$ & $\begin{array}{r}19 \\
10 \\
14 \\
2\end{array}$ & $\begin{array}{r}26 \\
15 \\
27 \\
4\end{array}$ & $\begin{array}{r}36 \\
26 \\
34 \\
8\end{array}$ & $\begin{array}{r}46 \\
38 \\
33 \\
9\end{array}$ & $\begin{array}{l}41 \\
19 \\
35 \\
13\end{array}$ \\
\hline \multicolumn{6}{|c|}{ Democrazia rappresentativa } \\
\hline $\begin{array}{l}\text { Portogallo } \\
\text { Spagna } \\
\text { Italia } \\
\text { Grecia }\end{array}$ & $\begin{array}{l}51 \\
35 \\
44 \\
37\end{array}$ & $\begin{array}{l}54 \\
50 \\
41 \\
47\end{array}$ & $\begin{array}{l}54 \\
53 \\
44 \\
57\end{array}$ & $\begin{array}{l}44 \\
45 \\
43 \\
57\end{array}$ & $\begin{array}{l}52 \\
70 \\
49 \\
65\end{array}$ \\
\hline \multicolumn{6}{|c|}{ Democrazia populista } \\
\hline $\begin{array}{l}\text { Portogallo } \\
\text { Spagna } \\
\text { Italia } \\
\text { Grecia }\end{array}$ & $\begin{array}{l}6 \\
9 \\
9 \\
2\end{array}$ & $\begin{array}{l}5 \\
9 \\
9 \\
2\end{array}$ & $\begin{array}{l}3 \\
6 \\
8 \\
2\end{array}$ & $\begin{array}{l}4 \\
4 \\
8 \\
2\end{array}$ & $\begin{array}{l}1 \\
2 \\
6 \\
4\end{array}$ \\
\hline \multicolumn{6}{|c|}{ Democrazia partecipativa } \\
\hline $\begin{array}{l}\text { Portogallo } \\
\text { Spagna } \\
\text { Italia } \\
\text { Grecia }\end{array}$ & $\begin{array}{l}23 \\
46 \\
33 \\
59\end{array}$ & $\begin{array}{l}14 \\
26 \\
24 \\
46\end{array}$ & $\begin{array}{r}7 \\
14 \\
13 \\
.34\end{array}$ & $\begin{array}{r}7 \\
12 \\
16 \\
31\end{array}$ & $\begin{array}{r}6 \\
9 \\
10 \\
18\end{array}$ \\
\hline
\end{tabular}

(vedi tab. 10). Com'era prevedibile, la legittimità dei partiti è elevata in tutti e quattro i paesi, soprattutto in Grecia, dove il $76 \%$ degli intervistati ha espresso un atteggiamento favorevole. All'estremo opposto troviamo il Portogallo, dove gli intervistati indifferenti erano più numerosi dei favorevoli. In Italia, dove la legittimità dei partiti è tutt'altro che elevata, $i$ giudizi positivi restano comunque molto più numerosi di quelli indifferenti $\mathrm{e}$ specie di quelli negativi. Il più basso favore per i partiti politici si riscontra, pertanto, in due paesi, Italia e Portogallo, i cui governi erano formati da coalizioni multipartitiche relativamente instabili e dove il desiderio di una «democrazia decisionista» era più forte. 
TAB. 10. Indice di legittimità partitica (valori percentuali)

\begin{tabular}{lcccc}
\hline Grado di legittimità & Portogallo & Spagna & Italia & Grecia \\
\hline Forte & 18 & 38 & 30 & 44 \\
Medio & 24 & 18 & 24 & 32 \\
Neutro & 44 & 30 & 32 & 18 \\
Nullo & 7 & 8 & 11 & 3 \\
Non so/nessuna risposta & 7 & 6 & 2 & 3 \\
Totale & 100 & 100 & 100 & 100 \\
\hline
\end{tabular}

TAB. 11. Correlazioni della legittimità partitica con la legittimità democratica e l'alienazione politica

\begin{tabular}{lcc}
\hline & Legittimità democratica & Alienazione politica \\
\hline Portogallo & 0,29 & $-0,35$ \\
Spagna & 0,45 & $-0,41$ \\
Italia & 0,28 & $-0,30$ \\
Grecia & 0,34 & $-0,20$ \\
\hline
\end{tabular}

Va sottolineato comunque che, in tutti e quattro i paesi, il grado di legittimità dei partiti è un po' inferiore alla legittimità specifica del regime democratico. Al fine di esplorare il rapporto tra legittimità dei partiti e legittimità del regime democratico, abbiamo calcolato i coefficienti di correlazione tra i due indici corrispondenti. La tab. 11 riporta i risultati, nonché le correlazioni tra l'indice di legittimità partitica e l'indice di alienazione politica (il quale, ricordiamo, attiene soprattutto alla percezione dell'efficacia dei singoli cittadini). I dati mostrano che la relazione tra favore per i partiti e legittimità democratica è più forte in Spagna, dove si è registrata anche la più forte relazione negativa tra alienazione politica e atteggiamenti nei confronti dei partiti.

La spiegazione più plausibile di questo risultato è che in Spagna i partiti hanno svolto un ruolo chiave nella riuscita della transizione democratica. Inoltre, al momento del sondaggio, il potente governo socialista era ancora molto popolare, e gli veniva riconosciuto un contributo decisivo al consolidamento del nuovo regime dopo il tentato golpe del 1981. Di converso, in Portogallo e Italia i partiti non vengono associati tanto alla democrazia quanto alla conflittualità, all'instabilità e al basso rendimento delle istituzioni. Di conseguenza, è essenziale chiedersi 
TAB. 12. Grado di simpatia per partiti, gruppi e istituzioni (punteggi medi ponderati, su una scala da 1 a 10)

\begin{tabular}{lcccc}
\hline & Portogallo & Spagna & Italia & Grecia \\
\hline Partiti & 4,4 & 4,2 & 4,1 & 4,9 \\
Sindacati & 4,5 & 4,3 & 4,5 & 7,9 \\
Gruppi di interesse & 5,3 & 5,7 & 5,6 & 5,4 \\
Chiesa & 7,0 & 5,4 & 6,7 & 7,3 \\
Istituzioni & 6,0 & 5,4 & 6,3 & 6,9 \\
Polizia & 5,8 & 6,1 & 7,1 & - \\
Forze armate & 6,3 & 5,2 & 6,4 & 6,7 \\
Magistratura & 5,8 & 4,9 & 5,5 & 7,1 \\
\hline
\end{tabular}

come mai i giudizi molto meno favorevoli nei confronti dei partiti in questi paesi non avessero riflessi negativi sulla legittimità della democrazia. Il tenue legame tra legittimità di regime e legittimità dei partiti (attestato dalle deboli correlazioni) indica che le fondamenta della legittimità democratica hanno a che fare più con le istituzioni che con i partiti.

Dal punto di vista della legittimità di regime, ciò può essere un bene, in quanto i partiti sono valutati meno positivamente di altre importanti istituzioni sociali e politiche. Su un termometro dei sentimenti con una scala da 1 a 10 , soltanto il $2 \%$ degli spagnoli e il $4 \%$ degli italiani hanno assegnato il punteggio massimo ai partiti; di converso, il 36\% degli spagnoli e il $45 \%$ degli italiani hanno espresso giudizi negativi (compresi tra 1 e 4). I partiti ricevono punteggi relativamente bassi persino in Grecia, dove il favore per la democrazia è particolarmente elevato. La tab. 12 riporta $i$ punteggi medi attribuiti ai partiti e ad altre istituzioni mediante un termometro dei sentimenti.

Per quale motivo vige questa ambiguità nei confronti dei partiti? Una risposta articolata a questa domanda esula dall'ambito di questo saggio in quanto richiederebbe un'analisi di un insieme molto ampio di dati. Tra le ipotesi più promettenti da controllare empiricamente dovremmo enumerare le seguenti: 1) le divisioni e la polarizzazione presupposte dal ruolo svolto in passato dai partiti in questi paesi; 2) la propaganda antipartitica intrapresa dai regimi autoritari del passato; 3) gli scandali relativi a casi di corruzione dei governi capeggiati da leader di partito; 4) il comportamento opportunista e divisivo delle élites partitiche, le quali sono ancora più visibili e invadenti nell'era televisiva; 5) l'occupazione dello stato e di settori della società ad 
opera dei partiti (almeno in Italia). In breve, $\mathrm{i}$ cittadini di questi paesi hanno forse adottato la posizione secondo la quale «ci rendiamo conto di aver bisogno dei partiti, ma non ci piacciono», ovvero «abbiamo bisogno dei partiti, ma poiché sappiamo troppe cose sul loro conto non ci piacciono» ${ }^{29}$.

\section{Alcune osservazioni conclusive}

Di fronte al «successo» degli ordinamenti democratici e al loro crescente radicamento nelle culture politiche dei quattro paesi in questione, le alternative politiche e le opposizioni al regime erano nel 1985 praticamente inesistenti. Gli atteggiamenti favorevoli alla democrazia sono diffusi fra tutti i gruppi sociali. Le uniche differenze sistematiche tra gli europei del Sud in termini di atteggiamenti verso la democrazia, di valutazione del passato e di percezione dell'efficacia delle istituzioni governative dipendono dall'autocollocazione sinistra-destra. Sotto questo profilo, a metà degli anni ottanta i regimi democratici dell'Europa del Sud possono ritenersi legittimi e consolidati.

$\mathrm{Si}$ sono registrate, tuttavia, notevoli differenze tra gli europei del Sud in merito alle concezioni di democrazia, come attesta il divario tra gli intervistati di destra, che preferiscono un governo decisionista, e quelli di sinistra, che esprimono una preferenza per ampie consultazioni dei gruppi sociali e per la partecipazione popolare diretta nei processi decisionali governativi. In ogni caso, una maggioranza schiacciante degli europei del Sud riconosce l'importanza del ruolo svolto dai partiti politici; e in ciascuno paese la forma di governo preferita è la «democrazia rappresentativa». Ciononostante $\mathrm{i}$ partiti vengono considerati inevitabili piuttosto che istituzioni sorrette da un elevato grado di simpatia popolare.

Insomma, questa rassegna degli atteggiamenti di fondo sottostanti al sostegno per la democrazia dà luogo a un quadro alquanto variegato. Da una parte, in tutti e quattro i paesi vi è una cultura politica realista, in cui il favore per la democrazia è forte, non si presentano alternative plausibili alla democrazia e i trascorsi autoritari suscitano nostalgia soltanto in una ridotta minoranza di persone. Allo stesso tempo sono particolarmente

\footnotetext{
${ }^{29}$ Questa osservazione è dovuta a un commento di Gianfranco Pasquino a una versione precedente di questo saggio.
} 
diffusi sentimenti di alienazione e di cinismo politico, una sfiducia intensa nelle élites, una limitata legittimità dei partiti e un basso livello di simpatia nei loro confronti (nonostante venga riconosciuto il loro ruolo politico).

Questo articolato percorso attraverso le dimensioni della cultura politica che più direttamente si collegano alla legittimità ci consente, oggi, di comprendere meglio le crescenti difficoltà politiche cui sono andati incontro i quattro paesi nel corso dei primi anni novanta, e che si sono richiamati all'inizio di questo saggio. Una menzione particolare merita, comunque, il caso italiano. Come si è visto, la vasta insoddisfazione per il funzionamento della democrazia e lo scarso consenso per i partiti attestano che l'Italia si trovava in una fase di potenziale mobilitazione elettorale. È pure il caso di sottolineare che quei dati erano stati rilevati a metà degli anni ottanta, in una fase cioè che, con il governo presieduto da Craxi, veniva quasi unanimemente considerata di riconsolidamento della democrazia italiana. Una fase, inoltre, in cui i problemi indotti dalla crisi economica non erano ancora percepiti e il debito pubblico cresceva nell'indifferenza, consapevole o colpevole, dei governanti.

Se si tiene conto, poi, della proporzionalità estrema del sistema elettorale all'epoca vigente e della successiva scomparsa del Pci, è possibile comprendere meglio il successo dei movimenti antisistema e antipartitici registrato alle elezioni regionali del 1990 e alle politiche di due anni dopo. Alla luce dei dati presentati in precedenza, il «terremoto» e la «svolta» del 1992 sono forse più correttamente interpretabili in termini di continuità con le caratteristiche di lungo periodo della cultura politica italiana. D'altra parte, l'ondata di protesta e di insoddisfazione che è venuta alla superficie in questi ultimi anni, se trae le sue origini da orientamenti da tempo rilevati dalle indagini demoscopiche, ha oggi raggiunto un'ampiezza tale da configurare l'Italia degli anni novanta come un caso - unico tra le democrazie dell'Europa meridionale - in cui la crisi di oggi assume i connotati della crisi di regime.

\section{Riferimenti bibliografici}

Agger, R.E., M.N. Goldstein e S.A. Pearl (1961), Political Cynicism: Measurement and Meaning, in «Journal of Politics», XXIII, pp. 477-506. 
Almond, G.A. e S. Verba (1963), The Civic Culture: Political Attitudes and Democracy in Five Nations, Princeton, Princeton University Press.

Arian, A. e M. Shamir (1983), The Primarily Political Functions of the Left-Right Continuum, in «Comparative Politics», XV, pp. 139158.

Bacalhau, M. (1978), Os Portugueses e a politica quatro anaos depois do 25 de april, Lisboa, Meseta.

Botella, J. (1992), La cultura politica en la España democrática, in R. Cotarelo (a cura di), Transición política y consolidación democrática. España (1975-1986), Madrid, Centro de Investigaciones Sociológicas, pp. 121-136.

Bruneau, T.C. (1983), People Support for Democracy in Post-revolutionary Portugal, in L.S. Graham e D.L. Wheeler (a cura di), Portugal: the Revolution and Its Consequences, Madison, University of Wisconsin Press, pp. 21-42.

- (1985), Continuity and Change in Portoguese Politics. Ten Years After the Revolution of 25 April 1974, in «West European Politics», VII, pp. 72-83.

Bruneau, T.C. e A. McLeod (1986), Politics in Contemporary Portugal: Parties and the Consolidation of Democracy, Boulder, Col., Lynn Reiner.

Citrin, J., H. McClosky, M. Shanks e P.M. Sniderman (1975), Personal and Political Sources of Political Alienation, in «British Journal of Political Science», V, pp. 1-31.

Condomines, J. e J.D. Barroso (1984), La dimension gauche-droite et la compétition entre les partis politiques en Europe du Sud (Portugal, Espagne, Grèce), in «Il Politico», IL, pp. 405-428.

Crozier, M.J., S.P. Huntington e J. Watanuki (1975), The Crisis of Democracy: Report on the Governability of Democracies to the Trilateral Commission, New York, New York University Press; trad. it. La crisi della democrazia, Milano, Franco Angeli, 1977.

Dahl, R. (1971), Poliarchy: Participation and Opposition, New Haven, Yale University Press; trad. it. Poliarchia. Partecipazione e opposizione nei sistemi politici, Milano, Franco Angeli, 1981.

Diamandouros, N. (1983), Greek Political Culture in Transition: Historical Origins, Evolution, Current Trends, in R. Clogg (a cura di), Greece in the 1980s, London, Macmillan, pp. 43-69.

Dimitras, P.E. (1987), Changes in Public Attitudes, in K. Featherstone e D.K. Katsoudas (a cura di), Political Change in Greece: Before and After the Colonels, London, Croon Helm, pp. 64-84.

- (1990), Greek Public Attitudes: Continuity and Change, in «International Journal of Public Opinion Research», II, pp. 92-115.

Di Palma, G. (1984), Government Performance: An Issue and Three Cases in Search of Theory, in G. Pridham (a cura di), The New Mediterranean Democracies: Regime Transition in Spain, Greece and Portugal, London, Frank Cass, pp. 172-187. 
- (1990), To Craft Democracies: Reflections on Democratic Transitions and Beyond, Berkeley, University of California Press.

Finifter, A. (1970), Dimensions of Political Alienation, in «American Political Science Review», LXIV, pp. 389-410.

- (1972), Alienation and the Political System, New York, John Wiley and Sons.

Fraser, J. (1971), Personal and Political Meaning and Correlates of Political Cynicism, in «Midwest Journal of Political Science», XV, pp. 347-364.

Gamson, W.A. (1968), Power and Discontent, Homewood, Dorsey.

Guidorossi, G. e G. Sani (1985), The Political Culture of Italy: Fragmentation, Isolation, Alienation from the Fifties to the Eighties, relazione presentata al XIII congresso dell'International Political Science Association, Paris.

Gunther, R. e J.R. Montero (1992), The Anchors of Partisanship: A Comparative Analysis of Voting Behavior in Four Southern European Democracies, non pubblicato.

Gunther, R., G. Sani e G. Shabad (1986), Spain After Franco: The Making of a Competitive Party System, Berkeley, University of California Press.

Heimer, F.W., J. Vala-Salvador e J.M. Leite Viegas (1989), Attitudes Towards Democracy in Contemporary Portugal, non pubblicato.

Kafetzis, P. (1988), Europe du Sud: à la recerche du citoyen et de la politique, non pubblicato.

King, A. (1975), Overload: Problems of Governing in the 1970's, in «Political Studies», XXIII, pp. 283-296.

Inglehart, R. e H.D. Klingemann (1976), Party Identification, Ideological Preference and the Left-Right Dimension among Western Mass Publics, in I. Budge, I. Crewe e D. Farlie (a cura di), Party Identification and Beyond: Representation of Voting and Party Competition, London, John Wiley and Sons.

Klingemann, H.D. (1987), Measuring Ideological Conceptualizations, in S.H. Barnes, M. Kaase et al. (a cura di), Political Action: Mass Participation in Five Western Democracies' Revisited: Spatial Models of Party Competition and Left-Right Measurements, in «International Political Science Review», VIII, pp. 333-342.

Laponce, J.A. (1981), Left and Right: The Topography of Political Perceptions, Toronto, University of Toronto Press.

Linz, J.J. (1974), The Consolidation of Regimes: $A$ Theoretical Problem Approach, relazione presentata all'International Studies Association World Congress, Toronto.

- (1978), Crisis, Breakdown and Requilibration, in J.J. Linz e A. Stepan (a cura di), The Breakdown of Democratic Regimes, Baltimore, Johns Hopkins University Press, pp. 14-124; trad. it. parziale La caduta dei regimi democratici, Bologna, Mulino, 1981.

- (1982), The Transitions from Authoritarian Regimes to Democratic 
Political Systems and the Problems of Consolidation of Political Democracy, intervento alla tavola rotonda organizzata dall'International Political Science Assocation, Tokyo.

- (1988), Legitimacy of Democracy and the Socio-economic System, in M. Dogan (a cura di), Comparing Pluralist Democracies, Boulder, Westview, pp. 65-113.

- (1989), I rapporti tra legittimazione ed efficacia di governo, in «Mondoperaio», n. 3, pp. 111-116.

- (1990), Transitions to Democracy, in «The Washington Quarterly», n. 1, pp. 143-164.

Linz, J.J., M. Gómez-Reino, F.A. Orizo, e D. Vila (1981), Informe sociológico sobre el cambio político en España, 1975-1981, Madrid, Euramérica.

Linz, J.J. e J.R. Montero (a cura di), (1986), Crisis y cambio: electores y partidos en la España de los Anos Ochenta, Madrid, Centro de Estudios Constitucionales.

Linz, J.J. e A. Stepan (1989), Political Crafting of Democratic Consolidation or Destruction: Europe and South American Comparisons, in R.A. Pastor (a cura di), Democracy in the Americas: Stopping the Pendulum, New York, Holmes and Meier, pp. 41-61.

- (1993), Democratic Transitions and Consolidation: Eastern Europe, Southern Europe and Latin America, New Haven, Yale University Press, in corso di stampa.

Lipset, S. (1959), Political Man: The Social Bases of Politics, Garden City, N.Y., Doubleday; trad. it. L'uomo e la politica, Milano, Comunità.

Llera, F.J. (1989), El sistema de partidos vasco: distancia ideológica y legitimación política, in «Revista española de investigaciones sociológicas», XXVIII, pp. 171-206.

- (1992), Conflicto en Euskadi' Revisited, in R. Gunther (a cura di), Politics, Society and Democracy: The Case of Spain, Boulder, Col., Westview Press.

Loewenberg, G. (1971), The Influence of Parliamentary Bebavior on Regime Stability, in «Comparative Politics», III, pp. 170-195.

Mannheimer, R. (1989), Una componente della decisione di voto: l'identificazione di partito, in Capire il voto. Contributi per l'analisi del comportamento elettorale in Italia, Milano, Franco Angeli, pp. 51-70.

Mannheimer, R. e G. Sani (1987a), Una componente della cultura politica: l'attaccamento al partito in quattro nazioni del Sud Europa, non pubblicato.

- (1987b), Il mercato elettorale. Identikit dell'elettore italiano, Bologna, Il Mulino.

Maravall, J.M. (1984), La política de la transición, Madrid, Taurus, $2^{\mathrm{a}}$ ed.

- (1991), Economic Reforms in New Democracies: The Southern Eu- 
ropean Experience, Madrid, Centro de Estudios Avanzados en Ciencias Sociales, Estudio/Working paper n. 22.

Maravall, J.M. e J. Santamaria (1986), Political Change in Spain and Prospects for Democracy, in G. O'Donnell et al. (1986), pp. 71-108.

Mattei, F. (1987), Le dimensioni dell'efficacia politica: aspetti metodologici, in «Rivista italiana di scienza politica», XVII, pp. 105-133.

Mavrogordatos, G.T. (1983), Rise of the Greek Sun: The Greek Elections of 1981, London, Centre for Contemporary Greek Studies, Occasional Paper n. 1.

McDonough, P., S.H. Barnes e A. López Pina (1986), The Growth of Democratic Legitimacy in Spain, in «American Political Science Review», LXXX, pp. 735-760.

Montero, J.R. (1986), Iglesia, secularización y comportamiento politico en España, in «Revista española de investigaciones sociológicas», XXXIV, pp. 131-159.

- (1992), Sobre la democracia en España: legitimidad, apoyos institucionales y significados, Madrid, Centro de Estudios Avanzados en Ciencias Sociales, Estudio/Working paper n. 39.

Montero, J.R. e M. Torcal (1990), Voters and Citizens in a New Democracy: Some Trend Data on Political Attitudes in Spain, in «International Journal of Public Opinion Research», II, pp. 116-140.

Morlino, L. (1980), Come cambiano i regimi politici. Strumenti di analisi, Milano, Franco Angeli.

- (1984), The Changing Relationship Between Parties and Society in Italy, in «West European Politics», VII, pp. 46-66.

Morlino, L. e F. Mattei (1992), Vecchio e nuovo autoritarismo nell'Europa mediterranea, in «Rivista italiana di scienza politica», XXII, pp. 137-160.

Muller, E.W. e T.O. Jukam (1977), On the Meaning of Political Support, in «American Political Science Review», LXXI, pp. 15611595.

O’Donnell, G., P.C. Schmitter e L. Whitehead (a cura di) (1986), Transitions from Authoritarian Rule: Southern Europe, Baltimore, Johns Hopkins University Press.

Papacosma, V.S. (1988), Politics and Culture in Greece, Ann Arbor, Center for Political Studies, University of Michigan.

Passeron, J.-C. (1982), Los silencios: contribución a la interpretación de las no respuestas en la encuesta de opinión, in «Revista española de investigaciones sociológicas», XVII.

Pérez Diaz, V. (1987), Iglesia y religión en la España contemporánea, in El retorno de la sociedad civil, Madrid, Instituto de Estudios Económicos, pp. 411-466.

Putnam, R.D. (1973), The Beliefs of Politicians: Ideology, Conflict and Democracy in Britain and Italy, New Haven, Yale University Press.

Richardson, B. (1990), The Development of Partisan Commitments in 
Post-Franquist Spain, Department of Political Science, Ohio State University.

Rose, R. (a cura di) (1980), Challenge to Governance, Beverly Hills, Sage.

Sani, G. (1974), A Test of a Least-Distance Model of Voting Choice: Italy 1972, in «Comparative Political Studies», VII, pp. 193-208.

- (1980), The Political Culture of Italy: Continuity and Change, in G.A. Almond e S. Verba (a cura di), The Civic Culture Revisited, Boston, Little, Brown and Co, pp. 273-321.

- (1981), Partiti e atteggiamenti di massa in Spagna e Italia, in «Rivista italiana di scienza politica», XI, pp. 235-279.

Sani, G. e J.R. Montero (1986), El espectro político: izquierda, derecha $y$ centro, in J.J. Linz e J.R. Montero (1986).

Sartori, G. (1987), The Theory of Democracy Revisited, Chatham, Chatham House.

Schaar, J. (a cura di) (1981), Legitimacy in the Modern State, New Brunswick, Transaction.

Shabad, G. (1994), Still the Exception? Democratization and Ethnic Nationalism in the Basque Country of Spain, in R. Gunther, G. Shabad, J.J. Linz, J.R. Montero e H.-J. Puhle, Electoral Change and Democratic Consolidation in Spain, in corso di stampa.

Schmitt, H. (1983), Party Government in Public Opinion: A European Cross-National Comparison, in «European Journal of Political Research», XI, pp. 353-376.

- (1989), On Party Attachment in Western Europe and the Utility of Eurobarometer Data, in «West European Politics», XII, pp. 122139.

- (1990), Party Attachment and Party Choice in the European Election of June 1989, in «International Journal of Public Opinion Research», II, pp. 169-181.

Schmitter, P.C. (1988), The Consolidation of Political Democracy in Southern Europe, non pubblicato.

Schwarts, D.C. (1973), Political Alienation and Political Behavior, Chicago, Aldine.

Van der Eijk, C. e B. Niemoller (1983), Theoretical and Methodological Considerations in the Use of Left-Right Scales, relazione presentata alle Joint Sessions dell'Ecpr, Salzburg.

Vila, D. e M. Gómez-Reino (1980), El proceso de cambio politico en el electorado (1973-1980), relazione presentata al seminario su «Electoral Surveys and Electoral Behavior», Madrid.

Wright, J.D. (1970), The Dissent of the Governed: Alienation and Democracy in America, New York, Academic Press. 